\title{
REFLEXIVITY AND ORDER PROPERTIES OF SCALAR-TYPE SPECTRAL OPERATORS IN LOCALLY CONVEX SPACES
}

BY

\author{
P. G. DODDS, B. de PAGTER ${ }^{1}$ AND W. RICKER ${ }^{2}$
}

\begin{abstract}
One of the principal results of the paper is that each scalar-type spectral operator in the quasicomplete locally convex space $X$ is reflexive. The paper also studies in detail the relation between the theory of equicontinuous spectral measures in locally convex spaces and the order properties of equicontinuous Bade complete Boolean algebras of projections.
\end{abstract}

0. Introduction. One of the principal results of this paper is that each scalar-type spectral operator $T$ in the quasicomplete, locally convex space $X$ is reflexive, i.e. the strongly closed subalgebra generated by the identity and $T$ in $\mathscr{L}(X)$, the space of continuous linear operators on $X$, consists precisely of those continuous linear operators on $X$ which leave invariant each (closed) $T$-invariant subspace of $X$. For the case that $X$ is a Banach space, this result was established by Gillespie [9] via an interesting factorization theorem in Banach function spaces, a method which does not appear to extend readily to the more general setting. The present approach, however, avoids factorization theorems by showing directly that each continuous linear functional on the strongly closed algebra generated by a Bade-complete, equicontinuous Boolean algebra of projections in $X$ has a representation of the form $\left\langle\cdot x, x^{\prime}\right\rangle$ for some $x \in X$ and $x^{\prime} \in X^{\prime}$, where $X^{\prime}$ denotes the dual space of $X$, a result which goes back to $R$. Pallu de la Barrière [20] for the case of Abelian von Neumann algebras in Hilbert space. Our method is based on ideas from the theory of Riesz spaces and yields considerable simplification of technique, even in the setting of Banach spaces.

The cornerstone of the present paper is the extension of the reflexivity theorem of Bade [2] to the setting of locally convex spaces proved in [6] via the theory of closed spectral measures and further refined and sharpened in [5] using purely intrinsic methods, based on order considerations. One of the new features which emerged from the approach of [5] was a type of "automatic continuity" theorem for a certain class of everywhere defined linear operators, even in the absence of a suitable closed-graph theorem. This idea is exploited in $\$ 1$ to show that an everywhere

\footnotetext{
Received by the editors December 10, 1984.

1980 Mathematics Subject Classification. Primary 47D30, 47B40; Secondary 28A60, 46A40.

Key words and phrases. Spectral operators, Boolean algebras, reflexivity, spectral measures, Riesz

${ }^{1}$ Research supported in part by a Visiting Research Fellowship at Flinders University.

${ }^{2}$ Research supported by Queen Elizabeth II Fellowship.
} spaces. 
defined scalar-type spectral operator is automatically continuous without some further assumption such as barreledness on the underlying space $X$. This yields further information concerning the structure of the space $L^{1}(P)$ of functions which are integrable with respect to a closed equicontinuous spectral measure $P$; in fact, if $P$ has a cyclic vector $x \in X$, then the space $L^{1}(P)$ consists precisely of those functions which induce multiplication operators on the space $L^{1}(P x)$ of functions which are integrable with respect to the closed point-evaluation measure $P x$. A simple consequence is an explicit representation, as a multiplier algebra, of the strongly closed algebra generated by an equicontinuous Bade $\sigma$-complete Boolean algebra of projections in $X$, a representation theorem due to Gillespie [8] for the case that $X$ is Banach, although, once again, the methods of [8] do not extend, even to metrizable spaces.

The second section contains a detailed study of order properties of closed algebras generated by Boolean algebras of projections and their associated cyclic spaces. The results presented in this section unify and systematically extend to locally convex spaces certain representation theorems familiar in the context of Banach spaces. In these representation theorems the theory of closed vector measures plays an efficient and decisive role. In the special situation that the underlying space $X$ is a complex Dedekind complete Riesz space with a complete locally solid Lebesgue topology, the strongly closed algebra generated by the Boolean algebra of the band projections in $X$ consists precisely of the continuous, order bounded and band preserving linear operators on $X$, a result which is not immediately apparent, but which leads to a purely order characterization of those spaces which can arise as the $L^{1}$-space of a closed equicontinuous spectral measure. The second section concludes with a characterization of those locally convex spaces $X$ which admit a Bade-complete, equicontinuous Boolean algebra of projections $\mathscr{M}$ with the property that the sum of finitely many cyclic subspaces is again cyclic. Such a severe restriction implies that the space $X$ must admit the structure of a locally solid Riesz space with strong order properties and it should be remarked that for such spaces, the reflexivity of scalar-type spectral operators is almost a direct consequence of the general form of the Bade reflexivity theory (see, for example, [5, Proposition 5.8]). It should be mentioned that a convenient tool used in this section is a complex form of the Freudenthal theorem, which is of independent interest.

The main reflexivity theorem is given in the third section and asserts that each unital closed subalgebra of the strongly closed algebra generated by a Bade complete Boolean algebra of projections is itself reflexive. This result, due to Gillespie [9] for the case that $X$ is Banach, immediately implies that each scalar-type spectral operator is a reflexive operator. The paper concludes with some remarks concerning the spectral resolution of a scalar-type spectral operator. A surprising example of a scalar-type spectral operator, with real spectrum, which has more invariant subspaces than its spectral resolution is given and the strongly closed subalgebra of $\mathscr{L}(X)$ generated by the (range of the) spectral resolution of a given scalar-type spectral operator is characterized as the smallest strongly closed unital inverse closed subalgebra of $\mathscr{L}(X)$ which contains $T$ and $T^{*}$. 
For basic results from the theory of Riesz spaces, the reader is referred to the monographs [1, 17, 18 and 30].

The authors wish to thank Igor Kluvanek for a number of interesting conversations, some of which were related to the subject matter of this paper.

1. Multiplier properties of integrable functions and cyclic spaces. In the first part of this section, we establish the notation to be used in the text and summarize those aspects of the theory of integration with respect to vector measures that are needed in the sequel; see [13] for a more comprehensive treatment. We then develop further some very special features of the theory of integration with respect to spectral measures. In particular we observe that for a given spectral measure $P$, each $P$-integrable function induces a multiplier operator in $L^{1}(P x), x \in X$, where $P x$ denotes the $X$-valued measure induced by $P$ via evaluation at the point $x$. Moreover, if $x$ is cyclic for $P$, then all multiplication operators on $L^{1}(P x)$ arise in this way.

It will always be assumed throughout this section that the locally convex Hausdorff space $X$ is quasicomplete and that $\mathscr{L}(X)$ is sequentially complete. The identity operator in $X$ is denoted by $I$. The adjoint of an operator $T \in \mathscr{L}(X)$ is denoted by $T^{\prime}$.

An $X$-valued vector measure is a $\sigma$-additive map $n: \Sigma \rightarrow X$ whose domain $\Sigma$ is a $\sigma$-algebra of subsets of a set $\Omega$. For each $x^{\prime} \in X^{\prime}$, the complex-valued measure $E \rightarrow\left\langle n(E), x^{\prime}\right\rangle, E \in \Sigma$, is denoted by $\left\langle n, x^{\prime}\right\rangle$. Its variation is denoted by $\left|\left\langle n, x^{\prime}\right\rangle\right|$, $x^{\prime} \in X^{\prime}$.

If $q$ is a continuous seminorm on $X$, let $U_{q}^{\circ}$ denote the polar of the closed unit ball of $q$. Then the $q$-semivariation of $n$ is the set function $q(n)$ given by

$$
q(n)(E)=\sup \left\{\left|\left\langle n, x^{\prime}\right\rangle\right|(E) ; x^{\prime} \in U_{q}^{\circ}\right\}, \quad E \in \Sigma .
$$

For each $E \in \Sigma$, the inequalities

(2) $\sup \{q(n(F)) ; F \in \Sigma, F \subseteq E\} \leqslant q(n) E \leqslant 4 \sup \{q(n(F)) ; F \in \Sigma, F \subseteq E\}$ hold [13, II, Lemma 1.2].

A complex-valued, $\Sigma$-measurable function $f$ on $\Omega$ is said to be $n$-integrable if it is integrable with respect to each measure $\left\langle n, x^{\prime}\right\rangle, x^{\prime} \in X^{\prime}$, and if, for every $E \in \Sigma$, there exists an element $\int_{E} f d n$ of $X$ such that

$$
\left\langle\int_{E} f d n, x^{\prime}\right\rangle=\int_{E} f d\left\langle n, x^{\prime}\right\rangle,
$$

for each $x^{\prime} \in X^{\prime}$. The map $f n: \Sigma \rightarrow X$ defined by

$$
(f n)(E)=\int_{E} f d n, \quad E \in \Sigma,
$$

is called the indefinite integral of $f$ with respect to $n$. The Orlicz-Pettis lemma implies that it is a vector measure. The element $(f n)(\Omega)=\int_{\Omega} f d n$ is denoted simply by $n(f)$.

The set of all $n$-integrable functions is denoted by $L(n)$. Members of $\Sigma$ are freely identified with their characteristic function. An $n$-integrable function is said to be $n$-null if its indefinite integral is the zero vector measure. Two $n$-integrable functions 
$f$ and $g$ are $n$-equivalent or equal $n$-almost everywhere (n-a.e.) if $|f-g|$ is $n$-null. A set $E \in \Sigma$ is $n$-null if $\chi_{E}=0, n$-a.e.

If $f$ is an $n$-integrable function, then for each continuous seminorm $q$ on $X$ we define the $q$-upper integral, $q(n)(f)$, by $q(n)(f)=q(f n)(\Omega)$. The function

$$
f \rightarrow q(n)(f), \quad f \in L(n),
$$

is then a seminorm on $L(n)$.

Denote by $\tau(n)$ the topology on $L(n)$ which is defined by the family of seminorms (3), for each continuous seminorm $q$ on $X$. The resulting locally convex space is not necessarily Hausdorff. The quotient space of $L(n)$ with respect to the subspace of all $n$-null functions is denoted by $L^{1}(n)$. The resulting Hausdorff topology on $L^{1}(n)$ is again denoted by $\tau(n)$. It is clear from (2) that $\tau(n)$ is the topology of uniform convergence on $\Sigma$ of indefinite integrals.

A vector measure $n: \Sigma \rightarrow X$ is said to be closed if the locally convex Hausdorff space $L^{1}(n)$ is complete. This agrees with the original definition given in [13]; see [25, Proposition 1].

If $W$ is an equicontinuous subset of $X^{\prime}$, then $q_{W}$ denotes the continuous seminorm on $X$ defined by

$$
q_{W}(x)=\sup \left\{\left|\left\langle x, x^{\prime}\right\rangle\right| ; x^{\prime} \in W\right\}, \quad x \in X .
$$

For $x \in X$ and an equicontinuous subset $W$ of $X^{\prime}$, let $q_{W}^{x}$ denote the continuous seminorm on $\mathscr{L}(X)$ given by

$$
q_{W}^{x}: T \rightarrow q_{w}(T x), \quad T \in \mathscr{L}(X),
$$

where $q_{W}$ is the seminorm (4). The collection of all such seminorms $q_{W}^{x}$ determines the topology of $\mathscr{L}(X)$.

Let $P: \Sigma \rightarrow \mathscr{L}(X)$ be an $\mathscr{L}(X)$-valued measure, with domain $\Sigma$ a $\sigma$-algebra of subsets of a set $\Omega$. For each $x \in X$, let $P x$ denote the $X$-valued vector measure

$$
P x: E \rightarrow P(E) x, \quad E \in \Sigma \text {. }
$$

Since the correspondence $\sum_{i} x_{i} \otimes x_{i}^{\prime} \rightarrow \xi \in(\mathscr{L}(X))^{\prime}$, defined by

$$
\xi: T \rightarrow \sum_{i}\left\langle T x_{i}, x_{i}^{\prime}\right\rangle, \quad T \in \mathscr{L}(X),
$$

is an (algebraic) isomorphism of the tensor product $X \otimes X^{\prime}$ onto the dual of $\mathscr{L}(X)$, it follows from the Orlicz-Pettis lemma that $P$ is $\sigma$-additive if and only if the complex-valued set function

$$
\left\langle P x, x^{\prime}\right\rangle: E \rightarrow\left\langle P(E) x, x^{\prime}\right\rangle, \quad E \in \Sigma,
$$

is $\sigma$-additive for each $x \in X$ and $x^{\prime} \in X^{\prime}$.

An operator-valued measure $P: \Sigma \rightarrow \mathscr{L}(X)$ always has bounded range in $\mathscr{L}(X)$. If the range of $P$, that is $P(\Sigma)=\{P(E) ; E \in \Sigma\}$, is an equicontinuous part of $\mathscr{L}(X)$, then $P$ is said to be equicontinuous.

A measure $P: \Sigma \rightarrow \mathscr{L}(X)$ is said to be a spectral measure if it is multiplicative and $P(\Omega)=I$. Of course, the multiplicativity of $P$ means that $P(E \cap F)=$ $P(E) P(F)$ for every $E, F \in \Sigma$. Since $\mathscr{L}(X)$ is a locally convex space, it is clear that spectral measures are vector measures. 
Proposition 1.1. Let $P: \Sigma \rightarrow \mathscr{L}(X)$ be an equicontinuous spectral measure and let $f$ be a P-integrable function. Let $x \in X$. Then for each Px-integrable function $g$ the function $\mathrm{fg}$ is also Px-integrable and has indefinite integral given by

$$
\int_{E} f g d P x=P(f)\left(\int_{E} g d P x\right), \quad E \in \Sigma .
$$

Furthermore, the associated linear map $m_{f}: L^{1}(P x) \rightarrow L^{1}(P x)$ defined by

$$
m_{f}: g \rightarrow f g, \quad g \in L^{1}(P x),
$$

is continuous.

Proof. Let $g_{n}=g \chi_{n}, n=1,2, \ldots$, where $\chi_{n}$ denotes the characteristic function of $\{w ;|g(w)| \leqslant n\}$. Then each function $g_{n}, n=1,2, \ldots$, is $P$-integrable [13, II, Lemma 3.1]. It follows that each function $\mathrm{fg}_{n} \in L^{1}(P), n=1,2, \ldots$, and

$$
\int_{E} f g_{n} d P=P(f) P\left(g_{n}\right) P(E)=P(f)\left(\int_{E} g_{n} d P\right)
$$

for every $E \in \Sigma[6$, Lemma 1.3].

Fix $x^{\prime} \in X^{\prime}$. It follows from (6) that

$$
\int_{E} f g_{n} d\left\langle P x, x^{\prime}\right\rangle=\int_{\Omega} g_{n} d\left\langle P x, P(f)^{\prime} P(E)^{\prime} x^{\prime}\right\rangle, \quad E \in \Sigma,
$$

for every $n=1,2, \ldots$ Since $g_{n} \rightarrow g$ pointwise and $g$ is $\left\langle P x, P(f)^{\prime} P(E)^{\prime} x^{\prime}\right\rangle$-integrable for each $E \in \Sigma$, the Dominated Convergence Theorem implies that

$$
\lim _{n \rightarrow \infty} \int_{E} f g_{n} d\left\langle P x, x^{\prime}\right\rangle=\int_{\Omega} g d\left\langle P x, P(f)^{\prime} P(E)^{\prime} x^{\prime}\right\rangle, \quad E \in \Sigma .
$$

It follows [14, Lemma 2.3] that $f g$ is $\left\langle P x, x^{\prime}\right\rangle$-integrable for each $x^{\prime} \in X^{\prime}$ and

$$
\int_{E} f g d\left\langle P x, x^{\prime}\right\rangle=\int_{\Omega} g d\left\langle P x, P(f)^{\prime} P(E)^{\prime} x^{\prime}\right\rangle, \quad E \in \Sigma .
$$

If $E \in \Sigma$, then certainly $P(f)\left(\int_{E} g d P x\right) \in X$. For each $x^{\prime} \in X^{\prime}$, we have (cf. [6, Proposition 1.7(iv)])

$$
\begin{aligned}
\left\langle P(f)\left(\int_{E} g d P x\right), x^{\prime}\right\rangle & =\left\langle\int_{E} g d P x, P(f)^{\prime} x^{\prime}\right\rangle=\left\langle\int_{\Omega} g d P x, P(f)^{\prime} P(E)^{\prime} x^{\prime}\right\rangle \\
& =\int_{\Omega} g d\left\langle P x, P(f)^{\prime} P(E)^{\prime} x^{\prime}\right\rangle .
\end{aligned}
$$

It is then clear from (7) that

$$
\left\langle P(f)\left(\int_{E} g d P x\right), x^{\prime}\right\rangle=\int_{E} f g d\left\langle P x, x^{\prime}\right\rangle, \quad x^{\prime} \in X^{\prime},
$$

for each $E \in \Sigma$. This shows that $f g$ is $P x$-integrable and has indefinite integral as stated in the proposition.

To prove the continuity of $m_{f}$, we recall (cf. (3)) that the topology of $L^{1}(P x)$ is specified by the seminorms

$$
g \rightarrow q(P x)(g)(\Omega), \quad g \in L^{1}(P x),
$$


for each continuous seminorm $q$ on $X$. So let $q$ be a continuous seminorm on $X$. Since

$$
q\left(P x\left(m_{f}(g)\right)\right)(\Omega)=\sup \left\{\left|\left\langle P x(f g), x^{\prime}\right\rangle\right|(\Omega) ; x^{\prime} \in U_{q}^{\circ}\right\}, \quad g \in L^{1}(P x),
$$

and the complex-measure $\left\langle(f g) P x, x^{\prime}\right\rangle$ is equal to $\left\langle g P x, P(f)^{\prime} x^{\prime}\right\rangle$ (cf. (8)), it follows that

$$
\begin{aligned}
q\left(P x\left(m_{f}(g)\right)\right)(\Omega) & \leqslant \sup \left\{\left|\left\langle P x(g), y^{\prime}\right\rangle\right|(\Omega) ; y^{\prime} \in U_{\hat{q}}^{\circ}\right\} \\
& =\hat{q}(P x(g))(\Omega)
\end{aligned}
$$

for each $g \in L^{1}(P x)$, where $\hat{q}$ is the continuous seminorm on $X$ corresponding to the equicontinuous subset $\left\{P(f)^{\prime} x^{\prime} ; x^{\prime} \in U_{q}^{\circ}\right\}$ of $X^{\prime}$. This shows $m_{f}$ is continuous and completes the proof of the proposition.

Since the constant function 1 is $P x$-integrable for each $x \in X$ [13, II, Lemma 3.1], it follows from Proposition 1.1 that if $f$ is a $P$-integrable function, then $f$ is also $P x$-integrable for each $x \in X$ and

$$
\int_{E} f d P x=P(f) P(E) x=P(E) P(f) x, \quad E \in \Sigma .
$$

That the converse to this statement also holds is the content of the following

Proposition 1.2. Let $P: \Sigma \rightarrow \mathscr{L}(X)$ be an equicontinuous spectral measure. If $f$ is a $\Sigma$-measurable function such that $f$ is $P x$-integrable for each $x \in X$, then $f$ is P-integrable.

Proof. Let $f_{n}=f \chi_{n}, n=1,2, \ldots$, where $\chi_{n}$ is the characteristic function of the set $\{w ;|f(w)| \leqslant n\}$. Define a linear operator $T_{f}: X \rightarrow X$ by

$$
T_{f}: x \rightarrow \int_{\Omega} f d P x, \quad x \in X .
$$

It follows that

$$
T_{f} x=\lim _{n \rightarrow \infty} \int_{\Omega} f_{n} d P x=\lim _{n \rightarrow \infty} P\left(f_{n}\right) x, \quad x \in X,
$$

where each operator $P\left(f_{n}\right)=\int_{\Omega} f_{n} d P, n=1,2, \ldots$, is continuous on $X$ (cf. [6, Proposition 1.7(iv)]).

It follows easily from the $\sigma$-additivity of $P$ that its range $P(\Sigma)$ is a strongly equicontinuous Boolean algebra of projections in the sense of [5, §3]. Since (9) implies that $T_{f}$ leaves invariant all closed subspaces of $X$ left invariant by each member of $P(\Sigma)$, it follows that $T_{f}$ is continuous [5, Corollary 5.7]. Finally, since $f$ is $\left\langle P x, x^{\prime}\right\rangle$-integrable for each $x \in X, x^{\prime} \in X^{\prime}$, and $T_{f} \in \mathscr{L}(X)$ satisfies

$$
\left\langle T_{f} x, x^{\prime}\right\rangle=\left\langle\int_{\Omega} f d P x, x^{\prime}\right\rangle=\int_{\Omega} f d\left\langle P x, x^{\prime}\right\rangle,
$$

Proposition 1.2 of [6] implies that $f$ is $P$-integrable.

We remark, that under additional assumptions on the underlying space $X$, Proposition 1.2 is well known, even for arbitrary operator-valued measures in $\mathscr{L}(X)$; see [15, Theorem 6.1], for example. In the case of spectral measures, Proposition 1.2 is therefore an improvement on previous results since no additional requirements on $X$ are needed. 
Let $\mathscr{M}$ be a commuting family of operators in $\mathscr{L}(X)$. For each $x \in X$, the cyclic subspace generated by $x$ with respect to $\mathscr{M}$ and denoted by $\mathscr{M}[x]$ is the smallest closed $\mathscr{M}$-invariant subspace of $X$ containing $x$. Of course, $\mathscr{M}[x]$ is the closure in $X$ of the linear span of $\{B x ; B \in \mathscr{M}\}$. A vector $x \in X$ such that $\mathscr{M}[x]=X$ is said to be cyclic with respect to $\mathscr{M}$. If $P$ is an $\mathscr{L}(X)$-valued spectral measure, then a vector $x \in X$ is said to be cyclic for $P$ if it is cyclic with respect to the range of $P$. In the presence of a cyclic vector the preceding proposition can be considerably sharpened (cf. [6, Corollary 4.9]).

Proposition 1.3. Let $P: \Sigma \rightarrow \mathscr{L}(X)$ be a closed, equicontinuous spectral measure. Suppose that $x \in X$ is a cyclic vector for $P$. If $f$ is a $\Sigma$-measurable fucntion such that $f g$ is $P x$-integrable whenever $g$ is $P x$-integrable, then $f$ is $P$-integrable and the linear map $m_{f}: L^{1}(P x) \rightarrow L^{1}(P x)$ defined by (5) is continuous.

Proof. To show that $f$ is $P$-integrable it suffices, by Proposition 1.2, to show that $f$ is $P y$-integrable for each $y \in X$. So fix $y \in X$. By Proposition 2.1 of [6] there is a $P x$-integrable function $h$ such that $y=P x(h)$. Then $P y$ is the vector measure

$$
P y: E \rightarrow P(E) P x(h)=P(E)\left(\int_{\Omega} h d P x\right), \quad E \in \Sigma ;
$$

see [6, Proposition 1.7(iv)]. It follows easily that for each $x^{\prime} \in X^{\prime}$, the measure $\left\langle P y, x^{\prime}\right\rangle$ is given by

$$
\left\langle P(E) y, x^{\prime}\right\rangle=\int_{E} h d\left\langle P x, x^{\prime}\right\rangle, \quad E \in \Sigma .
$$

Let $f_{n}, n=1,2, \ldots$, be defined as in the proof of Proposition 1.2. Then each function $f_{n}, n=1,2, \ldots$, is $\left\langle P y, x^{\prime}\right\rangle$-integrable for each $x^{\prime} \in X^{\prime}$ and it follows from (10) that

$$
\int_{E} f_{n} d\left\langle P y, x^{\prime}\right\rangle=\int_{E} h f_{n} d\left\langle P x, x^{\prime}\right\rangle, \quad E \in \Sigma,
$$

for each $x^{\prime} \in X^{\prime}$ and $n=1,2, \ldots$

Fix $x^{\prime} \in X^{\prime}$. Since $\left|h f_{n}\right| \leqslant|h f|, n=1,2, \ldots$, and $h f$ is $\left\langle P x, x^{\prime}\right\rangle$-integrable it follows from (11) and the Dominated Convergence Theorem that

$$
\lim _{n \rightarrow \infty} \int_{E} f_{n} d\left\langle P y, x^{\prime}\right\rangle=\int_{E} f h d\left\langle P x, x^{\prime}\right\rangle, \quad E \in \Sigma .
$$

Accordingly, $f$ is $\left\langle P y, x^{\prime}\right\rangle$-integrable and

$$
\int_{E} f d\left\langle P y, x^{\prime}\right\rangle=\int_{E} f h d\left\langle P x, x^{\prime}\right\rangle, \quad E \in \Sigma ;
$$

see, for example, [14, Lemma 2.3]. Since $P x(f h)$ is an element of $X$ (as $f h$ is $P x$-integrable) and satisfies

$$
\left\langle P x(f h), x^{\prime}\right\rangle=\left\langle\int_{\Omega} f h d P x, x^{\prime}\right\rangle=\int_{\Omega} f h d\left\langle P x, x^{\prime}\right\rangle=\int_{\Omega} f d\left\langle P y, x^{\prime}\right\rangle,
$$

for each $x^{\prime} \in X^{\prime}$, Proposition 1.7(iv) of [6] implies that $f$ is $P y$-integrable. Hence, $f$ is $P$-integrable. 
The continuity of $m_{f}$ is now an immediate consequence of Proposition 1.1. This completes the proof of the proposition.

Let $P: \Sigma \rightarrow \mathscr{L}(X)$ be a closed, equicontinuous spectral measure for which there exists a cyclic vector, say $x \in X$. Then each element $h$ of $L^{1}(P)$ induces a continuous linear map $m_{h}: L^{1}(P x) \rightarrow L^{1}(P x)$ defined by $m_{h}=m_{f}$, where $f$ is any representative of the equivalence class of $P$-integrable functions determining $h$ and $m_{f}$ is given by (5). Since $P$ and $P x$ have the same null sets (as $x$ is cyclic for $P$ ) it follows that $m_{h}$ is well defined. That is, the definition of $m_{h}$ is independent of the representative $f$. Furthermore, the family of operators

$$
M(P, x)=\left\{m_{h} ; h \in L^{1}(P)\right\}
$$

is a subalgebra of $\mathscr{L}\left(L^{1}(P x)\right)$; see [6, Lemma 1.3]. We equip $M(P, x)$ with the relative topology from $\mathscr{L}\left(L^{1}(P x)\right)$.

The main structure theorem for the cyclic space $X$, mentioned at the beginning of this section, can now be proved.

Proposition 1.4. Let $P: \Sigma \rightarrow \mathscr{L}(X)$ be a closed, equicontinuous spectral measure such that $P$ has a cyclic vector, say $x \in X$. Then the linear map

$$
\Phi_{x}: h \rightarrow m_{h}, \quad h \in L^{1}(P),
$$

is an algebraic and topological isomorphism of the complete locally convex algebra $L^{1}(P)$ onto the algebra $M(P, x)$ of multiplier operators in $L^{1}(P x)$. In particular, $M(P, x)$ is a complete subalgebra of $\mathscr{L}\left(L^{1}(P x)\right)$.

Proof. To show $\Phi_{x}$ is injective, suppose that $f$ and $g$ are $P$-integrable functions such that $m_{f}=m_{g}$. Then $m_{f}(1)=m_{g}(1)$ implies that $f=g$ in $L^{1}(P x)$, and, hence, that

$$
(P(f)-P(g)) x=\int_{\Omega}(f-g) d P x=0 .
$$

Since $x$ is cyclic for $P$ it follows that $P(f)=P(g)$ and, hence, that $f=g$ in $L^{1}(P)$ [6, Proposition 1.2].

To show the continuity of $\Phi_{x}$ we recall that the continuous seminorms determining the topology of $\mathscr{L}\left(L^{1}(P x)\right)$ are of the form

$$
T \rightarrow q(P x)(T g), \quad T \in \mathscr{L}\left(L^{1}(P x)\right),
$$

where $g \in L^{1}(P x)$ and $q$ is a continuous seminorm on $X$ (cf. (3), (4)). Accordingly, if we denote the element $\int_{\Omega} g d P x$ of $X$ by $y$, then it follows from (2) that

$$
\begin{aligned}
q(P x)\left(\Phi_{x}(f) g\right) & =q(P x)(f g) \leqslant 4 \sup \left\{q\left(\int_{E} f g d P x\right) ; E \in \Sigma\right\} \\
& =4 \sup \left\{q\left(\left(\int_{E} f d P\right) y\right) ; E \in \Sigma\right\} \\
& =4 \sup \left\{q^{y}\left(\int_{E} f d P\right) ; E \in \Sigma\right\} \leqslant 4 q^{y}(P)(f),
\end{aligned}
$$

for each $f \in L^{1}(P)$. Since $q^{y}(P)(\cdot)$ is a continuous seminorm on $L^{1}(P)$, this shows that $\Phi_{x}$ is continuous. 
To verify the continuity of $\Phi_{x}^{-1}$ let $m_{f_{\alpha}} \rightarrow 0$ in $M(P, x)$, that is, $h f_{\alpha} \rightarrow 0$ in $L^{1}(P x)$ for each $h \in L^{1}(P x)$. By Proposition 1.5 of [6], to show that $\Phi_{x}^{-1}\left(m_{f_{\alpha}}\right)=f_{\alpha}$ $\rightarrow 0$ in $L^{1}(P)$ it suffices to show that $P\left(f_{\alpha}\right) \rightarrow 0$ in $\mathscr{L}(X)$, that is, $\int_{\Omega} f_{\alpha} d P y \rightarrow 0$ in $X$ for $y \in X$. So, let $y \in X$. Since $x$ is cyclic for $P$ there is $h \in L^{1}(P x)$ such that $y=\int_{\Omega} h d P x$ [6, Proposition 2.1]. An argument similar to that used to deduce (12) implies that

$$
\int_{\Omega} f_{\alpha} d P y=\int_{\Omega} h f_{\alpha} d P x
$$

for each $\alpha$. Since $h f_{\alpha} \rightarrow 0$ in $L^{1}(P x)$ it follows that $\int_{\Omega} h f_{\alpha} d P x \rightarrow 0$ in $X$ [6, Proposition 2.1], and hence (13) implies that $\int_{\Omega} f_{\alpha} d P y \rightarrow 0$ in $X$, as required.

Finally, to complete the proof, we note that $L^{1}(P)$ is a complete, commutative locally convex algebra with identity [6, Proposition 1.4].

Corollary 1.5. Let $P$ and $x \in X$ be as in Proposition 1.4. Then there is $a$ bicontinuous linear isomorphism $\mathscr{U}$ of $L^{1}(P x)$ onto $X$ such that

$$
\mathscr{U}\left(m_{f} g\right)=P(f) \mathscr{U} g
$$

for all $f \in L^{1}(P)$ and $g \in L^{1}(P x)$.

Proof. The proof follows immediately from Proposition 1.4 and the fact that the integration map $\mathscr{U}$ defined by

$$
\mathscr{U}: g \rightarrow \int_{\Omega} g d P x, \quad g \in L^{1}(P x),
$$

is a bicontinuous isomorphism of $L^{1}(P x)$ onto $X$ [6, Proposition 2.1].

We remark that Corollary 1.5 can be viewed as a natural extension to the locally convex setting of a well-known representation theorem for cyclic Banach spaces due to T. A. Gillespie [8]. Indeed, if $X$ is a Banach space, then the $P$-integrable functions are precisely the $P$-essentially bounded functions [7, XVIII, Theorem 2.11(c)], that is, $L^{1}(P)$ and $L^{\infty}(P)$ are equal as sets in which case we observe that Corollary 1.5 is essentially Theorem 3.5 of [8], with the function space $L^{1}(P x)$ playing the role of the normed Köthe space $L_{\rho}^{a}$ in [8]. However, it should be noted that the proof in [8] is based on the existence of a "Bade functional" for $P$, a technique which is not available in the locally convex setting, even if the space $X$ is metrizable, as has been pointed out by B. Walsh [29, p. 315]. It is for this reason that the space of functions $L_{\rho}^{a}$ in [8] can be taken with respect to a real-valued measure on $\Omega$ whereas, in the case of locally convex spaces, it is not possible in general to replace the space $L^{1}(P x)$, where $P x$ is a vector measure, by a function space with respect to some scalar-valued measure.

2. Order properties and representation theorems. In this section, we investigate the order structure of those subspaces of the quasicomplete locally convex Hausdorff space $X$ which are cyclic with respect to a complete Boolean algebra of projections, using the theory of closed vector measures as one of our principal tools. As a by-product of independent interest, our approach yields explicit representation theorems which exhibit certain classes of locally convex Riesz spaces as $L^{1}$-spaces of closed vector measures. In what follows, we shall assume familiarity with the basic notions of the theory of Riesz spaces as set out in the monographs [1, 18 and 30]. 
Let $\mathscr{M}$ be an equicontinuous Boolean algebra of projections in $X$. We will assume that $\mathscr{M}$ is Bade complete, that is, $\mathscr{M}$ is complete as an abstract Boolean algebra and whenever $\left\{B_{\alpha}\right\} \subseteq M$ is downwards filtering to 0 in $\mathscr{M}$, it follows that $B_{\alpha} \rightarrow 0$ in $\mathscr{L}(X)$. Let $\mathscr{M}$ be displayed as the range of a closed, equicontinuous spectral measure $P$ on the Borel subsets $\Sigma$ of the Stone space $\Omega$ of $\mathscr{M}$. For each continuous seminorm $q$ on $X$ and $x \in X$, set $\rho_{q}(x)=q(P x)(\Omega)$. It follows from [13, II, Lemma 2.2] that

$$
\rho_{q}(x)=\sup \left\{q(P(f)(x)) ; f \in L^{1}(P), 0 \leqslant|f| \leqslant 1\right\} .
$$

From the equicontinuity of $\mathscr{M}$, it follows that $\left\{P(f): f \in L^{1}(P), 0 \leqslant|f| \leqslant 1\right\}$ is again an equicontinuous part of $\mathscr{L}(X)$ and from this it follows that the topology on $X$ defined by the system of seminorms $\left\{\rho_{q}: q\right.$ a continuous seminorm on $\left.X\right\}$, which we shall denoted simply by $\left\{\rho_{q}\right\}$, is precisely the given topology on $X$. Given $x \in X$, it is clear that

$$
\rho_{q}\left(\int_{\Omega} f d P x\right)=\rho_{q}\left(\int_{\Omega}|f| d P x\right), \quad f \in L^{1}(P x) .
$$

Moreover, if $f, g \in L^{1}(P x)$ and $0 \leqslant f \leqslant g$, then it is also clear that

$$
\rho_{q}\left(\int_{\Omega} f d P x\right) \leqslant \rho_{q}\left(\int_{\Omega} g d P x\right) .
$$

Let now $x \in X$ be given. Then the integration map

$$
P x: f \rightarrow(P x) f=\int_{\Omega} f d P x, \quad f \in L^{1}(P x),
$$

is a topological isomorphism of $L^{1}(P x)$ onto the cyclic space $\mathscr{M}[x]$; see [6, Proposition 2.1]. Accordingly, we can induce an order structure on $\mathscr{M}[x]$ from that of $L^{1}(P x)$ as follows: if $y \in \mathscr{M}[x]$, define $y \geqslant 0$ if and only if $(P x)^{-1}(y) \geqslant 0$. Since $P x$ is a closed measure [6, Proposition 1.7(iii)], the complex Riesz space $L^{1}(P x)$ is Dedekind complete [13, III, Theorem 4.2], and consequently the integration map $P x$ induces on $\mathscr{M}[x]$ the structure of a complex, Dedekind complete Riesz space. From (14) and (15), it follows that each seminorm $\rho_{q}$ is a Riesz seminorm on $\mathscr{M}[x]$ so that the topology defined on the complex Riesz space $\mathscr{M}[x]$ by the system of Riesz seminorms $\left\{\rho_{q}\right\}$ is locally solid. It is not difficult to see that the order structure on $\mathscr{M}[x]$ defined above coincides with that defined directly in [5] so that it follows from Corollary 4.13 of [5] that if in addition $x$ is a cyclic vector for $\mathscr{M}$, then the Boolean algebra $\mathscr{M}$ may be identified with the Boolean algebra of all band projections on the Riesz space $\mathscr{M}[x]=X$. This fact may also be seen directly by considering the form of the band projections on $L^{1}(P x)$. Since $x=\int_{\Omega} 1 d P x$, it follows that $x$ is a weak order unit in $\mathscr{M}[x]$.

Combining the preceding remarks, we obtain the following sharpening of Proposition 2.1 of [6]. We recall that a locally solid Riesz space $L$ is said to have Lebesgue topology if whenever $\left\{u_{\alpha}\right\} \subseteq L$ is downwards filtering to 0 in $L$, it follows that $u_{\alpha} \rightarrow 0$ for the given topology on $L$. 
Proposition 2.1. Let $\mathscr{M}$ be an equicontinuous, Bade complete Boolean algebra of projections in $X$, displayed as the range of a closed, equicontinuous spectral measure $P$. If $x \in X$, then the integration map

$$
P x: L^{1}(P x) \rightarrow \mathscr{M}[x]
$$

induces on $\mathscr{M}[x]$ the structure of a Dedekind complete, locally solid Riesz space with Lebesgue topology in which $x$ is a weak order unit. The integration map Px is then a Riesz isomorphism as well as a topological isomorphism. If, in addition, $x$ is a cyclic vector for $\mathscr{M}$, then $\mathscr{M}$ may be identified with the Boolean algebra of all band projections on the Riesz space $\mathscr{M}[x]=X$.

We make some remarks concerning the preceding result. It is well known that a Banach space which is cyclic with respect to a (Bade) $\sigma$-complete Boolean algebra of projections can be given an equivalent norm and an order structure with respect to which it is a Banach lattice with an order continuous norm and weak order unit. This was shown by A. Veksler [27] and is a special case of the preceding Proposition 2.1. Using ideas from the theory of normed Köthe spaces, a more concrete representation was obtained by T. A. Gillespie [8]. For quasicomplete locally convex spaces, the family of seminorms $\left\{\rho_{q}\right\}$ was introduced by B. Walsh [29] who showed that each subspace of $X$ which is cyclic with respect to an equicontinuous spectral measure has the structure of a Dedekind complete, complex Riesz space with a locally solid Lebesgue topology. The preceding proposition, however, yields additional information in the form of an explicit representation theorem, achieved by utilizing the theory of closed vector measures.

Before proceeding to our next result, we recall the notion of a complex $f$-algebra. The (real) Riesz space $\mathscr{A}$ is called a Reisz algebra if $\mathscr{A}$ is an algebra as well as a Riesz space with the additional property that $u v \geqslant 0$ for all $0 \leqslant u, v \in \mathscr{A}$. The Riesz algebra $\mathscr{A}$ is called an $f$-algebra if $u \wedge v=0$ in $\mathscr{A}$ implies that $(w u) \wedge v=(u w) \wedge$ $v=0$ for all $0 \leqslant w \in \mathscr{A}$. For general properties of $f$-algebras, a convenient reference is Chapter 20 of [30]. It is to be noted in particular that any Archimedean $f$-algebra is commutative. An algebra which is the complexification of a (real) Archimedean $f$-algebra is called a complex f-algebra (see $[4, \S 5])$. Necessarily, a complex $f$-algebra is commutative.

Now let $\langle\mathscr{M}\rangle$ denote the closed subalgebra of $\mathscr{L}(X)$ generated by the equicontinuous, Bade complete Boolean algebra $\mathscr{M}$. If $\mathscr{M}$ is displayed as the range of a closed, equicontinuous spectral measure $P$, then the space $L^{1}(P)$ has the structure of a Dedekind complete, complex Riesz space. If we assume further that $\mathscr{L}(X)$ is sequentially complete, then it follows from Lemma 1.3 of [6] that $L^{1}(P)$ is in fact a complex $f$-algebra in which multiplication is (separately) continuous, as follows from Proposition 1.1. By Proposition 1.5 of [6], the integration map $P: f \mapsto P(f)$, $f \in L^{1}(P)$, is a topological algebra isomorphism of $L^{1}(P)$ onto $\langle\mathscr{M}\rangle$. We apply now considerations similar to those contained in Proposition 2.1. By $\{q\}$, we denote the family of continuous seminorms on $X$. 
Proposition 2.2. Let $\mathscr{M}$ be an equicontinuous, Bade complete Boolean algebra of projections in the quasicomplete space $X$ and let $P$ be the associated spectral measure on the Stone space $\Omega$ of $\mathscr{M}$. If $\mathscr{L}(X)$ is sequentially complete, then the integration map

$$
P: f \mapsto P(f)=\int_{\Omega} f d P, \quad f \in L^{1}(P),
$$

induces on $\langle\mathscr{M}\rangle$ the structure of a Dedekind complete, complex f-algebra with separately continuous multiplication. With respect to this order structure, the topology defined on $\langle\mathscr{M}\rangle$ by the seminorms $\left\{\rho_{q}(\cdot x) ; x \in X ; q \in\{q\}\right\}$ coincides with the relative $\mathscr{L}(X)$-topology and is locally solid, complete and Lebesgue. The integration map $P$ is a topological and f-algebra isomorphism of $L^{1}(P)$ onto $\langle\mathscr{M}\rangle$. The identity operator $I$ is a weak order unit for $\langle\mathscr{M}\rangle$ and the Boolean algebra of principal components of $I$ in the complex Riesz space $\langle\mathscr{M}\rangle$ coincides with the Boolean algebra $\mathscr{M}$.

We remark that the order structure on $\langle\mathscr{M}\rangle$ given by the preceding proposition coincides with that defined in [5].

In order to prove the next result we need an abstract polar decomposition for elements in a Dedekind complete complex Riesz space. The sign-function that will appear in such a decomposition theorem is, however, not an element of the Riesz space itself, but is a linear operator on the space. We recall some relevant notions. Let $L$ be a complex Riesz space. We denote the real part of $L$ by $\operatorname{Re} L$, so that $L=\operatorname{Re} L+i \operatorname{Re} L$ (see e.g. [30, §91]). Two elements $f$ and $g$ in $L$ are called disjoint, denoted by $f \perp g$, if $|f| \wedge|g|=0$. A linear mapping $\pi$ from $L$ into itself is called band preserving if $\pi f \perp g$ whenever $f \perp g$ in $L$. An order bounded, band preserving mapping in $L$ is called an orthomorphism. The space of all orthomorphisms in $L$ is denoted by $\operatorname{Orth}(L)$. With respect to composition as multiplication, $\operatorname{Orth}(L)$ is a complex $f$-algebra, with the identity operator as unit element. Moreover, $\operatorname{Re} \operatorname{Orth}(L)$ can be identified with $\operatorname{Orth}(\operatorname{Re} L)$. For the general theory of orthomorphisms in Riesz spaces we refer to [30, Chapter 20], and for properties of orthomorphisms on complex Riesz spaces we refer to [5, §2]. In particular we recall that for any $\pi \in \operatorname{Orth}(L)$ and $f \in L$ we have $|\pi f|=|\pi| f||=|\pi||f|$. Furthermore, if $\pi=\operatorname{Re} \pi+i \operatorname{Im} \pi$ in $\operatorname{Orth}(L)$, and if we denote $\bar{\pi}=\operatorname{Re} \pi-i \operatorname{Im} \pi$, then $\pi \bar{\pi}=|\pi|^{2}$.

Proposition 2.3. Let $L$ be a Dedekind $\sigma$-complete complex Riesz space.

(i) For any $f \in L$ there exist $\sigma \in \operatorname{Orth}(L)$ such that $\sigma f=|f|$ and $|\sigma|=I$.

(ii) If $f, g \in L$ with $|g| \leqslant|f|$, then there exists $\pi \in \operatorname{Orth}(L)$ such that $g=\pi f$ and $|\pi| \leqslant I$.

Proof. (i) If $0 \leqslant v \leqslant u$ in $\operatorname{Re} L$, then an application of the Freudenthal spectral theorem yields an element $\pi \in \operatorname{Orth}(\operatorname{Re} L)$ such that $0 \leqslant \pi \leqslant I$ and $\pi u=v$ (for the details of the proof see [17, Theorem 7.6]). Now assume that $|g| \leqslant u$ with $g$, $u \in \operatorname{Re} L$. Then $0 \leqslant g^{+} \leqslant u$ and $0 \leqslant g^{-} \leqslant u$, so there exist $\pi_{1}, \pi_{2} \in \operatorname{Orth}(\operatorname{Re} L)$, $0 \leqslant \pi_{1}, \pi_{2} \leqslant I$, such that $\pi_{1} u=g^{+}$and $\pi_{2} u=g^{-}$. Putting $\pi=\pi_{1}-\pi_{2}$ we get $\pi u=g$. 
Now take $f \in L$. It follows from the inequalities $|\operatorname{Re} f| \leqslant|f|$ and $|\operatorname{Im} f| \leqslant|f|$ that there exist $\sigma_{1}, \sigma_{2} \in \operatorname{Orth}(\operatorname{Re} L)$ such that $\operatorname{Re} f=\sigma_{1}|f|$ and $\operatorname{Im} f=\sigma_{2}|f|$. Defining $\boldsymbol{\sigma}=\sigma_{1}-i \sigma_{2}$ gives that $\bar{\sigma}|f|=\left(\sigma_{1}+i \sigma_{2}\right)|f|=f$. Note that $|\boldsymbol{\sigma}\|f|=| \bar{\sigma}|f \|=| f \mid$, and so

$$
\sigma f=\sigma \bar{\sigma}|f|=|\sigma|^{2}|f|=|\sigma|(|\sigma||f|)=|\sigma||f|=|f| .
$$

Hence, the orthomorphism $\sigma$ satisfies $\sigma f=|f|$. Since $|\sigma||f|=|f|$, we can modify $\sigma$ outside the band generated by $f$ such that $|\sigma|=I$.

(ii) Suppose $|g| \leqslant|f|$ in $L$. By (i) there exist $\pi_{1}, \pi_{2} \in \operatorname{Orth}(L)$, with $\left|\pi_{1}\right|=\left|\pi_{2}\right|=I$, such that $f=\pi_{1}|f|$ (and hence $\left.\bar{\pi}_{1} f=|f|\right)$ and $g=\pi_{2}|g|$. Furthermore, there exists $\pi_{3} \in \operatorname{Orth}(L), 0 \leqslant \pi_{3} \leqslant I$, such that $|g|=\pi_{3}|f|$. Hence

$$
g=\pi_{2}|g|=\pi_{2} \pi_{3}|f|=\pi_{2} \pi_{3} \bar{\pi}_{1} f
$$

so $\pi=\pi_{2} \pi_{3} \bar{\pi}_{1}$ is the desired orthomorphism.

Proposition 2.4. Let $X$ be a Dedekind complete, complex Riesz space with locally solid, Lebesgue topology. Assume that $X$ is quasicomplete and let $\mathscr{M}$ be the Boolean algebra of band projections in $X$.

(i) $X$ is topologically complete and $\mathscr{M}$ is an equicontinuous, Bade complete Boolean algebra of projections in $X$.

(ii) The closed $\mathscr{M}$-invariant subspaces of $X$ are precisely the bands in $X$.

(iii) The algebraic sum of any finite number of closed, $\mathscr{M}$-invariant subspaces in $X$ is closed.

(iv) $\operatorname{Orth}(X) \cap \mathscr{L}(X)$ is an order ideal in $\operatorname{Orth}(X)$ and coincides with $\langle\mathscr{M}\rangle$.

Assume, in addition, that $\mathscr{L}(X)$ is sequentially complete.

(v) The complex f-algebra $\operatorname{Orth}(X)$ coincides with $\langle\mathscr{M}\rangle$.

(vi) If $X$ has weak order unit $e \geqslant 0$, then there exists a closed, equicontinuous $\mathscr{L}(X)$-valued spectral measure $P$ with range $\mathscr{M}$, for which $e$ is a cyclic vector and such that the integration map Pe is a topological and Riesz isomorphism of $L^{1}(P e)$ onto $X$.

Proof. (i) That $X$ is topologically complete is a direct consequence of $[\mathbf{5}$, Proposition 2.3]. If $q$ is any Riesz seminorm defining the topology of $X$, observe that

$$
q(E x)=q(|E x|)=q(E|x|) \leqslant q(|x|)=q(x)
$$

for all $E \in \mathscr{M}$ and all $x \in X$. It follows that $\mathscr{M}$ is an equicontinuous Boolean algebra of projections in $X$. Since $X$ is Dedekind complete and the given topology on $X$ is Lebesgue, it follows that $\mathscr{M}$ is even Bade complete.

(ii) Since the topology on $X$ is Lebesgue, the bands in $X$ are precisely the closed order ideals in $X$. Now, it is clear that each band in $X$ is closed and $\mathscr{M}$-invariant and consequently, it suffices to show that each closed $\mathscr{M}$-invariant subspace of $X$ is an order ideal of $X$. This, however is contained in Proposition 2.3 since the orthomorphisms $\sigma, \pi$ exhibited there clearly belong to $\langle\mathscr{M}\rangle$.

(iii) That the sum of finitely many closed $\mathscr{M}$-invariant subspaces of $X$ is closed is now a simple consequence of the fact that the sum of finitely many projection bands is again a projection band [18, Theorem 30.1].

(iv) If $\sigma, \pi \in \operatorname{Orth}(X)$ with $0 \leqslant|\sigma| \leqslant|\pi|$, it follows from

$$
0 \leqslant|\sigma|(|x|) \leqslant|\pi|(|x|)=|\pi(x)|, \quad x \in X
$$


that if $\pi$ belongs to $\mathscr{L}(X)$, then so does $\sigma$ and hence the continuous orthomorphisms on $X$ form an order ideal in $\operatorname{Orth}(X)$. Since the closed $\mathscr{M}$-invariant subspaces of $X$ are precisely the bands in $X$ by (ii), it follows from Corollary 5.6 of [5] that $\operatorname{Orth}(X) \cap \mathscr{L}(X) \subseteq\langle\mathscr{M}\rangle$. Conversely, if $T \in\langle\mathscr{M}\rangle$, then $T$ is band-preserving and hence order bounded by Lemma 2.6 of [5]. Consequently $T \in \mathscr{L}(X) \cap$ $\operatorname{Orth}(X)$, and by this the proof of (iv) is complete.

(v) The proof of (v) is almost identical to (iv) by an appeal to Corollary 5.7 rather than Corollary 5.6 of [5].

(vi) Let $\mathscr{M}$ be displayed as the range of an equicontinuous, closed spectral measure $P$ on the Stone space of $\mathscr{M}$, which is possible from (i). The well-known theorem of Freudenthal then asserts that the weak unit $e$ is cyclic for $P$. The proof of (vi) now follows directly from Proposition 2.1, after noting that the given order structure on $X$ in fact coincides with that induced by the integration map $P e$.

It has been noted earlier that if $P$ is a closed equicontinuous spectral measure, then $L^{1}(P)$ is a Dedekind complete, complex unital $f$-algebra with separately continuous multiplication and a complete, locally solid Lebesgue topology. It is not without interest to remark that these properties actually characterise those spaces which can arise as $L^{1}$-spaces of closed, equicontinuous spectral measures. In fact, let $\mathscr{A}$ be a Dedekind complete, complex unital $f$-algebra with complete locally solid Lebesgue topology for which multiplication is (separately) continuous. If $A \in \mathscr{A}$, define $m_{A} \in \mathscr{L}(\mathscr{A})$ via $m_{A}(B)=A B, B \in \mathscr{A}$. The map $A \mapsto m_{A}, A \in \mathscr{A}$, is then a topological and $f$-algebra isomorphism of $\mathscr{A}$ onto $\operatorname{Orth}(\mathscr{A})$, which is a complete linear subspace of $\mathscr{L}(\mathscr{A})$. From (iv) of Proposition 2.4 , it follows that $\operatorname{Orth}(\mathscr{A})$ coincides with $\langle\mathscr{M}\rangle$, where $\mathscr{M}$ is the complete Boolean algebra of band projections in $\mathscr{A}$. If $P$ is the associated spectral measure on the Stone space of $\mathscr{M}$, then $P$ is a closed, equicontinuous spectral measure. The given order structure on $\operatorname{Orth}(\mathscr{A})$ coincides with that induced on $\langle\mathscr{M}\rangle$ by the integration map, which therefore induces a topological and f-algebra isomorphism of $L^{1}(P)$ onto $\operatorname{Orth}(\mathscr{A})$ by Proposition 2.2, and hence of $L^{1}(P)$ onto $\mathscr{A}$.

We summarize these remarks in the following

Proposition 2.5. If $\mathscr{A}$ is a Dedekind complete complex unital f-algebra with a complete locally solid Lebesgue topology for which multiplication is separately continuous, then there exists a closed equicontinuous spectral measure $P$ and a topological and f-algebra isomorphism of $L^{1}(P)$ onto $\mathscr{A}$.

It is an immediate consequence of Proposition 2.4(ii) and Proposition 2.1, that if $\mathscr{M}$ is an equicontinuous, Bade complete Boolean algebra of projections in the (arbitrary) quasicomplete space $X$ and if $x \in X$ is cyclic for $\mathscr{M}$, then each closed $\mathscr{M}$-invariant subspace of $X$ is again cyclic. In particular, the algebraic sum of finitely many cyclic subspaces of a cyclic space, being closed by Proposition 2.4(iii), is again cyclic. In general, for spaces $X$ which do not have a cyclic vector with respect to a given Boolean algebra $\mathscr{M}$, the property that the algebraic sum of finitely may cyclic subspaces is always cyclic imposes strong restrictions on the space $X$ and the Boolean algebra $\mathscr{M}$. Before indicating the nature of these restrictions, we give 
several equivalent formulations of the property that the algebraic sum of finitely many cyclic subspaces is again cyclic. For the case of Banach spaces, the following result is due to C. Rall [21, Satz 5.1]. The proof given in [21] remains valid in the locally convex setting due to Propositions 2.1 and 2.4, so the details are omitted.

We recall first that if $\mathscr{M}$ is a Bade-complete Boolean algebra of projections in $X$ and if $x \in X$, then the carrier of $x$ in $\mathscr{M}$ is defined to be inf $\{E \in \mathscr{M}: E x=x\}$.

Proposition 2.6. Let $X$ be a quasicomplete space for which the space $\mathscr{L}(X)$ is sequentially complete and let $\mathscr{M} \subseteq \mathscr{L}(X)$ be an equicontinuous, Bade complete Boolean algebra of projections. Then the following statements are equivalent.

(i) The system $\{\mathscr{M}[x] ; x \in X\}$ of cyclic subspaces of $X$ is upwards filtering with respect to inclusion.

(ii) The algebraic sum of finitely many $\mathscr{M}$-cyclic subspaces of $X$ is cyclic.

(iii) For each $x \in X$, there exists a projection $E \in \mathscr{M}$ with range equal to $\mathscr{M}[x]$, in which case $E$ is precisely the carrier of $x$ in $\mathscr{M}$.

We now determine the structure of those spaces $X$ which admit an equicontinuous, Bade complete Boolean algebra $\mathscr{M}$ of projections for which one (and hence all) of the preceding equivalent conditions holds. We say in this case that the space $X$ is quasicyclic with respect to $\mathscr{M}$. The following description of quasicyclic spaces is due to C. Rall [21] for the case that $X$ is Banach. While our proof follows the general lines of Satz 5.5 in [21], it is based on the representation described in Proposition 2.1 .

Proposition 2.7. Let $\mathscr{M}$ be an equicontinuous, Bade complete Boolean algebra of projections in the quasicomplete space $X$ with $\mathscr{L}(X)$ sequentially complete. If $X$ is quasicyclic with respect to $\mathscr{M}$, then $X$ is topologically complete and there exists in $X$ the structure of a complex Riesz space such that $X$ is a locally solid complete Riesz space which is Dedekind complete, has Lebesgue topology and is such that the Boolean algebra of band projections coincides with the Boolean algebra $\mathscr{M}$.

Proof. Let $\left\{Q_{\alpha}\right\}$ be a maximal disjoint system of carrier projections in $\mathscr{M}$, corresponding to the elements $\left\{x_{\alpha}\right\}$ in $X$. By Proposition 2.6, $\mathscr{M}\left[x_{\alpha}\right]$ is the range of $Q_{\alpha}$, so in particular, $\mathscr{M}\left[x_{\alpha}\right] \cap \mathscr{M}\left[x_{\beta}\right]=\{0\}$ whenever $\alpha \neq \beta$. Let $Y=\oplus_{\alpha} \mathscr{M}\left[x_{\alpha}\right]$. The maximality of the system $\left\{Q_{\alpha}\right\}$ implies that $\sup _{\alpha} Q_{\alpha}=I$ in $\mathscr{M}$, hence $Y$ is dense in $X$. By Proposition 2.1 each $\mathscr{M}\left[x_{\alpha}\right]$ has the structure of a Dedekind complete complex Riesz space such that the induced topology in $\mathscr{M}\left[x_{\alpha}\right]$ is a complete Lebesgue topology.

Clearly, $Y=\oplus_{\alpha} \mathscr{M}\left[x_{\alpha}\right]$ is a Riesz space with respect to the direct sum ordering. Now observe that $\mathscr{M}\left[x_{\alpha_{1}}\right] \oplus \cdots \oplus \mathscr{M}\left[x_{\alpha_{n}}\right]=\mathscr{M}\left[x_{\alpha_{1}}+\cdots+x_{\alpha_{n}}\right]$ holds for any finite collection $\alpha_{1}, \ldots, \alpha_{n}$. Moreover, the direct sum Riesz space structure in $\mathscr{M}\left[x_{\alpha_{1}}\right] \oplus \cdots \oplus \mathscr{M}\left[x_{\alpha_{n}}\right]$ coincides with the Riesz space structure defined by Proposition 2.1 on $\mathscr{M}\left[x_{\alpha_{1}}+\cdots+x_{\alpha_{n}}\right]$. From this observation it is clear that $Y$ has the preLebesgue property. Using [5, Propositions 2.2 and 2.3], it follows that $X$ is the topological completion of $Y$ and that the Riesz space structure of $Y$ extends to $X$, such that $X$ is a Dedekind complete, locally solid, complex Riesz space with Lebesgue topology, by Proposition 10.5 of [1]. 
It remains to identify the Boolean algebra of band projections in $X$ with $\mathscr{M}$. By the continuity of the lattice operations it follows that $0 \leqslant B x \leqslant x$ for all $0 \leqslant x \in X$ and all $B \in \mathscr{M}$, and hence $B$ is a band projection in $X$ [18, Theorem 24.5]. In particular, for each index $\alpha, \mathscr{M}\left[x_{\alpha}\right]$ is a projection band in $X$ with band projection $Q_{\alpha}$, and, by [5, Corollary 4.12], the band projections in the Riesz space $\mathscr{M}\left[x_{\alpha}\right]$ coincide with the Boolean algebra $\left\{B Q_{\alpha}: B \in \mathscr{M}\right\}$. Now let $Q$ be a band projection in $X$. For each $\alpha, Q Q_{\alpha}$ is a band projection in $\mathscr{M}\left[x_{\alpha}\right]$, and hence $Q Q_{\alpha} \in \mathscr{M}$. Now it follows from $Q=\sum_{\alpha} Q Q_{\alpha}$ that $Q \in \mathscr{M}$. By this, the proof is complete.

We remark that the class of quasicyclic spaces properly includes the class of cyclic spaces. Indeed, from Proposition 2.4, examples are provided by taking for $X$ any complex Dedekind complete Riesz space, with a complete Lebesgue locally solid topology but which does not possess a weak order unit.

We conclude this section with some remarks concerning the structure of $\langle\mathscr{M}\rangle$ if $\mathscr{L}(X)$ is not assumed sequentially complete. Once again, let $\mathscr{M}$ be an equicontinuous Bade complete Boolean algebra of projections in the quasicomplete space $X$ and let $\langle\mathscr{M}\rangle$ be the closed subalgebra of $\mathscr{L}(X)$ generated by $\mathscr{M}$. As observed in Proposition 2.2 , if $\mathscr{L}(X)$ is assumed sequentially complete, then $\langle\mathscr{M}\rangle$ has the structure of a Dedekind complete, complex $f$-algebra such that the relative $\mathscr{L}(X)$ topology in $\langle\mathscr{M}\rangle$ is complete, locally solid and Lebesgue. Without sequential completeness of $\mathscr{L}(X)$, it is still true that $\langle\mathscr{M}\rangle$ has the structure of a Dedekind complete, complex $f$-algebra with locally solid Lebesgue topology, but $\langle\mathscr{M}\rangle$ is not in general topologically complete. Indeed, in this more general situation, the closed algebra $\langle\mathscr{M}\rangle^{\#}$ generated by $\mathscr{M}$ in the space $\mathscr{L}^{\sharp}(X)$ of all linear operators on $X$ (equipped with the topology of pointwise convergence on $X$ ) is a Dedekind complete $f$-algebra with a complete, locally solid Lebesgue topology (see [5, Propositions 3.6 and 3.12]). Clearly, $\langle\mathscr{M}\rangle$ is a subalgebra of $\langle\mathscr{M}\rangle^{\#}$. We assert that $\langle\mathscr{M}\rangle$ is even an order ideal in $\langle\mathscr{M}\rangle^{\#}$, from which follow the stated properties of $\langle\mathscr{M}\rangle$. To this end, suppose that $|S| \leqslant|T|$ in $\langle\mathscr{M}\rangle^{\#}$ with $T \in\langle\mathscr{M}\rangle$. An application of Proposition 2.3 in the $f$-algebra $\langle\mathscr{M}\rangle^{\#}$ yields an operator $R \in\langle\mathscr{M}\rangle^{\#}$ with $|R| \leqslant I$ such that $S=R T$. It follows from [5, Proposition 3.11] that $R \in\langle\mathscr{M}\rangle$ and hence $S \in\langle\mathscr{M}\rangle$.

3. Reflexive subalgebras and scalar-type spectral operators. Let $X$ be a quasicomplete, locally convex Hausdorff space and suppose that $N$ is a closed subalgebra of $\mathscr{L}(X)$ containing $I$. The subalgebra $N$ is called reflexive if $N$ consists precisely of those operators in $\mathscr{L}(X)$ which leave invariant each closed $N$-invariant subspace of $X$. An operator $T \in \mathscr{L}(X)$ will be called reflexive if the closed subalgebra of $\mathscr{L}(X)$ generated by $T$ and $I$ is reflexive. The main purpose of the present section is to show that every scalar-type spectral operator in the sense of $N$. Dunford [7] is a reflexive operator.

Now let $\mathscr{M}$ be an equicontinuous Bade complete Boolean algebra of projections in $X$ and let $\langle\mathscr{M}\rangle$ be the strongly closed algebra generated by $\mathscr{M}$.

The main result of [5] asserts that $\langle\mathscr{M}\rangle$ is a reflexive subalgebra of $\mathscr{L}(X)$. This was also shown in [6] under the additional hypothesis that $\mathscr{L}(X)$ is sequentially complete. Of course, for the case that $X$ is Banach, this result is due to W. Bade [2]. We will show now that closed unital subalgebras of $\langle\mathscr{M}\rangle$ are reflexive. 
We start with the following lemma, which is well known for Banach spaces; see [3 or 9, Lemma 4.5]. Its extension to the locally convex setting presents no difficulties.

LEMMA 3.1 (CF. [9, LeMMA 4.5]). Let $N$ be a closed unital subalgebra of $\mathscr{L}(X)$, and let $T \in \mathscr{L}(X)$ be given.

(i) $T$ leaves invariant every $N$-invariant subspace of $X$ if and only if $\left\langle T x, x^{\prime}\right\rangle=0$ whenever $x \in X$ and $x^{\prime} \in X^{\prime}$ are such that $\left\langle S x, x^{\prime}\right\rangle=0$ for all $S \in N$.

(ii) $T \in N$ if and only if $\sum_{i=1}^{n}\left\langle T x_{i}, x_{i}^{\prime}\right\rangle=0$ whenever $x_{1}, \ldots, x_{n} \in X$ and $x_{1}^{\prime}, \ldots, x_{n}^{\prime}$ $\in X^{\prime}$ are such that $\sum_{i=1}^{n}\left\langle S x_{i}, x_{i}^{\prime}\right\rangle=0$ for all $S \in N$.

Suppose that $N$ is a closed unital subalgebra of $\langle\mathscr{M}\rangle$, and assume that $T \in \mathscr{L}(X)$ leaves invariant all closed $N$-invariant subspaces of $X$. Then it follows from the extension of the Bade reflexivity theorem to locally convex spaces [5] that $T \in\langle\mathscr{M}\rangle$. The above lemma shows that in order to prove that $N$ is reflexive, it is sufficient to show that whenever $x_{1}, \ldots, x_{n} \in X$ and $x_{1}^{\prime}, \ldots, x_{n}^{\prime} \in X^{\prime}$ are given, there exist elements $x \in X$ and $x^{\prime} \in X^{\prime}$ such that

$$
\sum_{i=1}^{n}\left\langle S x_{i}, x_{i}^{\prime}\right\rangle=\left\langle S x, x^{\prime}\right\rangle
$$

for all $S \in\langle\mathscr{M}\rangle$. In the next part of this section we will prove that this is indeed the case.

We introduce some notation. Given $x \in X$ and $x^{\prime} \in X^{\prime}$, we will denote (formally) by $x \otimes x^{\prime}$ the linear functional on $\langle\mathscr{M}\rangle$ defined by

$$
\left(x \otimes x^{\prime}\right)(T)=\left\langle T x, x^{\prime}\right\rangle, \quad T \in\langle\mathscr{M}\rangle .
$$

Let $\langle\mathscr{M}\rangle^{\prime}$ denote the topological dual of $\langle\mathscr{M}\rangle$ (with respect to the strong operator topology). As is well known, $\langle\mathscr{M}\rangle^{\prime}$ consists precisely of all functionals of the form

$$
\sum_{i=1}^{n} x_{i} \otimes x_{i}^{\prime}, \quad x_{1}, \ldots, x_{n} \in X, x_{1}^{\prime}, \ldots, x_{n}^{\prime} \in X^{\prime}, n \in \mathbf{N} .
$$

In this notation, to guarantee the existence of elements $x \in X$ and $x^{\prime} \in X^{\prime}$ such that (16) is valid, it suffices to prove the following

Proposition 3.2. Given $x_{1}, \ldots, x_{n} \in X$ and $x_{1}^{\prime}, \ldots, x_{n}^{\prime} \in X^{\prime}$, there exist $x \in X$ and $x^{\prime} \in X^{\prime}$ such that

$$
\sum_{i=1}^{n} x_{i} \otimes x_{i}^{\prime}=x \otimes x^{\prime}
$$

in $\langle\mathscr{M}\rangle^{\prime}$. Equivalently, any element of the dual space of $\langle\mathscr{M}\rangle$ is of the form $x \otimes x^{\prime}$ for appropriate $x \in X$ and $x^{\prime} \in X^{\prime}$.

The proof of this proposition, which will be divided into five lemmas, makes use of the theory of Riesz spaces. First we introduce some relevant notions. Let $L$ denote an (Archimedean) complex Riesz space. As usual, $L_{\text {denotes the order dual }}$ of $L$ (see [30, §83]). A linear functional $\varphi \in L^{\tilde{L}}$ is called order continuous if $\inf \left|\varphi\left(u_{\alpha}\right)\right|=0$ whenever $u_{\alpha} \downarrow 0$ in $L$. The set of all order continuous linear functionals on $L$ is denoted by $\tilde{L_{n}} ; \tilde{L_{n}}$ is a band in $\tilde{L^{\sim}}[30,887]$. Given $0 \leqslant \varphi \in \tilde{L}$ the ideal 
$N_{\varphi}=\{f \in L: \varphi(|f|)=0\}$ is called the null ideal of $\varphi$. The disjoint complement $C_{\varphi}=N_{\varphi}^{d}$ is called the carrier of $\varphi$. Note that $\varphi$ is strictly positive on $C_{\varphi}$, i.e., that $\varphi(u)=0$ with $0 \leqslant u \in C_{\varphi}$ implies that $u=0$. Now assume that $L$ is Dedekind complete and take $0 \leqslant \varphi \in \tilde{L_{n}}$. Then $N_{\varphi}$ is a band and $L=C_{\varphi} \oplus N_{\varphi}$. Moreover, if $0 \leqslant \varphi, \psi \in \tilde{L_{n}}$, then $\varphi \wedge \psi=0$ is equivalent to $C_{\varphi} \perp C_{\psi}$ (i.e., $C_{\varphi} \cap C_{\psi}=\{0\}$ ).

The following lemma is one of the important tools in proving Proposition 3.2.

LEMMA 3.3 (COMPLEX BOUNDED RADON - NikODYM THEOREM). Let L be a Dedekind complete complex Riesz space and suppose that $|\psi| \leqslant|\varphi|$ in $L_{n}^{\sim}$. Then there exists an orthomorphism $\pi$ in L such that $\psi=\varphi \circ \pi$ and $|\pi| \leqslant I$.

Proof. For any $\pi \in \operatorname{Orth}(L)$ let $\pi^{\sim}: L_{n}^{\sim} \rightarrow L_{n}^{\sim}$ be defined by $\pi^{\sim}(\lambda)=\lambda \circ \pi$, $\lambda \in L_{n}^{\tilde{n}}$. Then $\pi^{\sim}$ is an orthomorphism in $L_{n}^{\sim}$. Moreover, the mapping $\pi \mapsto \pi^{\sim}$ is clearly a positive algebra homomorphism from the $f$-algebra $\operatorname{Orth}(L)$ into the $f$-algebra $\operatorname{Orth}\left(L_{n}^{-}\right)$, and hence, being disjointness preserving, is a Riesz homomorphism. Therefore, $|\lambda \circ \pi|=|\lambda| \circ|\pi|$ for all $\lambda \in L_{n}^{\sim}$ and $\pi \in \operatorname{Orth}(L)$. Using this observation, the result of the lemma follows from the corresponding result for real spaces [30, Theorem 145.2], analogous to the proof of Proposition 2.3(i).

Before returning to spectral operators we prove one more general result concerning Riesz spaces which will be useful.

Lemma 3.4. Let $L$ be a Riesz space with the principal projection property and let $\mathscr{J}$ be a subset of the positive cone $L^{+}$of $L$ such that:

(i) $u \in \mathscr{J}$ implies that $\alpha u \in \mathscr{J}$ for all $\alpha \geqslant 0$.

(ii) If $u \in L$ and $v \in \mathscr{J}$ with $0 \leqslant u \leqslant v$, then $u \in \mathscr{J}$.

(iii) If $u, v \in \mathscr{J}$ and $u \wedge v=0$, then $u+v \in \mathscr{J}$.

Then $u+v \in \mathscr{J}$ whenever $u, v \in \mathscr{J}$.

Proof. Take $u, v \in \mathscr{J}$. Let $P$ be the band projection onto the band $\left\{(u-v)^{+}\right\}^{d d}$. Then $P\left((u-v)^{-}\right)=0$, so $P u-P v \geqslant 0$. This implies that $0 \leqslant P v \leqslant P u \leqslant u$, and hence $0 \leqslant P(u+v) \leqslant 2 u$. By (i) we have $2 u \in \mathscr{J}$, and so it follows from (ii) that $P(u+v) \in \mathscr{J}$. Similarly, it follows from $(I-P)\left((u-v)^{+}\right)=0$ that

$$
(I-P)(u+v) \in \mathscr{J} \text {. }
$$

Since $[P(u+v)] \wedge[(I-P)(u+v)]=0$, it follows from (iii) that $u+v=$ $P(u+v)+(I-P)(u+v) \in \mathscr{J}$, as required.

Note that the above lemma applies in particular if the Riesz space $L$ is Dedekind complete.

Now we return to the closed algebra $\langle\mathscr{M}\rangle$ generated by the equicontinuous Bade complete Boolean algebra $\mathscr{M}$ in $\mathscr{L}(X)$. As before, $\langle\mathscr{M}\rangle^{\prime}$ denotes the topological dual of $\langle\mathscr{M}\rangle$. Since $\langle\mathscr{M}\rangle$ is a complex Riesz space, the order dual $\langle\mathscr{M}\rangle^{\sim}$ is defined, and since the topology on $\langle\mathscr{M}\rangle$ is locally solid, $\langle\mathscr{M}\rangle^{\prime}$ is an order ideal in $\langle\mathscr{M}\rangle^{\tilde{}}$ [1, Theorem 5.7]. Moreover, since the topology on $\langle\mathscr{M}\rangle$ is a Lebesgue topology, it follows that $\langle\mathscr{M}\rangle^{\prime} \subseteq\langle\mathscr{M}\rangle_{n}$. Let

$$
\mathscr{K}=\left\{\varphi \in\langle\mathscr{M}\rangle^{\prime}: \varphi=x \otimes x^{\prime} \text { for some } x \in X, x^{\prime} \in X^{\prime}\right\} .
$$


We have to show that $\mathscr{K}=\langle\mathscr{M}\rangle^{\prime}$. It is sufficient to show that $\mathscr{K}$ is a linear subspace of $\langle\mathscr{M}\rangle^{\prime}$.

LEMMA 3.5. $\mathscr{K}$ is a solid subset of $\langle\mathscr{M}\rangle^{\prime}$, i.e., if $|\psi| \leqslant|\varphi|$ in $\langle\mathscr{M}\rangle^{\prime}$, and $\varphi \in \mathscr{K}$, then $\psi \in \mathscr{K}$.

Proof. Suppose that $\varphi, \psi \in\langle\mathscr{M}\rangle^{\prime}$, with $|\psi| \leqslant|\varphi|$ and $\varphi \in \mathscr{K}$. Then $\varphi=x \otimes x^{\prime}$ for some $x \in X$ and $x^{\prime} \in X^{\prime}$. Since $\langle\mathscr{M}\rangle^{\prime} \subseteq\langle\mathscr{M}\rangle_{n}$, we can apply Lemma 3.3, so there exists $\pi \in \operatorname{Orth}(\langle\mathscr{M}\rangle)$ such that $\psi=\varphi \circ \pi,|\pi| \leqslant I$. Since $\langle\mathscr{M}\rangle$ is a unital $f$-algebra, there exists $A \in\langle\mathscr{M}\rangle$ such that $\pi(T)=T A$ for all $T \in\langle\mathscr{M}\rangle[30$, Theorem 141.1]. Hence $\psi(T)=\left(x \otimes x^{\prime}\right)(T A)=\left\langle T A x, x^{\prime}\right\rangle=\left((A x) \otimes x^{\prime}\right)(T)$ for all $T \in\langle\mathscr{M}\rangle$. This shows that $\psi=(A x) \otimes x^{\prime} \in \mathscr{K}$.

Before stating the next lemma, we recall that any band projection in the $f$-algebra $\langle\mathscr{M}\rangle$ is given by multiplication by an idempotent. Moreover, the idempotents in $\langle\mathscr{M}\rangle$ are precisely the projections in $\mathscr{M}$ [5, Proposition 4.4]. Hence, the band projections in $\langle\mathscr{M}\rangle$ are given by multiplications by elements of $\mathscr{M}$.

LemMA 3.6. If $0 \leqslant \varphi, \psi \in \mathscr{K}$ and $\varphi \wedge \psi=0$ in $\langle\mathscr{M}\rangle^{\prime}$, then $\varphi+\psi \in \mathscr{K}$.

Proof. Let $x, y \in X$ and $x^{\prime}, y^{\prime} \in X^{\prime}$ be such that $\varphi=x \otimes x^{\prime}$ and $\psi=y \otimes y^{\prime}$. By the remarks prior to Lemma 3.6, the band projection in $\langle\mathscr{M}\rangle$ onto the carrier $C_{\varphi}$ of $\varphi$ is given by multiplication by a projection $E \in \mathscr{M}$. Similarly, let the band projection onto $C_{\psi}$ be given by multiplication with $F \in \mathscr{M}$. It follows from $\varphi \wedge \psi=0$ that $C_{\varphi} \perp C_{\psi}$, and so $E F=0$.

Let $E^{\prime}: X^{\prime} \rightarrow X^{\prime}$ denote the adjoint of $E$. Now it follows from $\varphi(T)=\varphi(T E)$ and from $T E=E T E$ that

$$
\varphi(T)=\varphi(E T E)=\left\langle T E x, E^{\prime} x^{\prime}\right\rangle=\left((E x) \otimes\left(E^{\prime} x^{\prime}\right)\right)(T)
$$

for all $T \in\langle\mathscr{M}\rangle$. Hence $\varphi=(E x) \otimes\left(E^{\prime} x^{\prime}\right)$. Similarly we get $\psi=(F y) \otimes\left(F^{\prime} y^{\prime}\right)$. Now observe that

$$
\left((E x) \otimes\left(F^{\prime} y^{\prime}\right)\right)(T)=\left\langle F T E x, y^{\prime}\right\rangle=\left\langle T E F x, y^{\prime}\right\rangle=0
$$

for all $T \in\langle\mathscr{M}\rangle$, so $(E x) \otimes\left(F^{\prime} y^{\prime}\right)=0$. In like manner, we find that $(F y) \otimes\left(E^{\prime} x^{\prime}\right)$ $=0$. This implies that

$$
(E x+F y) \otimes\left(E^{\prime} x^{\prime}+F^{\prime} y^{\prime}\right)=(E x) \otimes\left(E^{\prime} x^{\prime}\right)+(F y) \otimes\left(F^{\prime} y^{\prime}\right),
$$

and hence $\varphi+\psi \in \mathscr{K}$.

LEMMA 3.7. If $\varphi, \psi \in \mathscr{K}$, then $\varphi+\psi \in \mathscr{K}$.

Proof. Let $\mathscr{J}$ be the set of all positive elements in $\mathscr{K}$ and let $L=\langle\mathscr{M}\rangle^{\prime}$. Clearly, $\mathscr{J}$ satisfies condition (i) of Lemma 3.4. By Lemma 3.5, $\mathscr{J}$ satisfies condition (ii) and by the above lemma condition (iii) is satisfied as well. Hence, by Lemma 3.4, $\varphi+\psi \in \mathscr{J}$ whenever $\varphi, \psi \in \mathscr{J}$, i.e. $\mathscr{K}$ is closed for addition of positive elements. Now, suppose that $\varphi, \psi \in \mathscr{K}$ are arbitrary. By Lemma 3.5, it follows that $|\varphi|,|\psi| \in \mathscr{K}$ and so $|\varphi|+|\psi| \in \mathscr{K}$. It now follows from the inequality $|\varphi+\psi| \leqslant|\varphi|$ $+|\psi|$ and from a further application of Lemma 3.5 that $\varphi+\psi \in \mathscr{K}$. 
We have thus shown that the set $\mathscr{K}$ is a linear subspace of $\langle\mathscr{M}\rangle^{\prime}$, and this completes the proof of Proposition 3.2. We are now in a position to prove the main result of this section.

THEOREM 3.8. Let $\mathscr{M}$ be an equicontinuous Bade complete Boolean algebra of projections in the quasicomplete space $X$ and let $\langle\mathscr{M}\rangle$ be the closed subalgebra of $\mathscr{L}(X)$ generated by $\mathscr{M}$. Each unital closed subalgebra of $\langle\mathscr{M}\rangle$ is a reflexive subalgebra of $\mathscr{L}(X)$.

Proof. Let $N$ be a closed unital subalgebra of $\langle\mathscr{M}\rangle$, and suppose that $T \in \mathscr{L}(X)$ leaves invariant all closed $N$-invariant subspaces of $X$. By the reflexivity theorem [5, Corollary 5.6], it follows that $T \in\langle\mathscr{M}\rangle$. A combination of Lemma 3.1 and Proposition 3.2 now yields the desired result.

An operator $T \in \mathscr{L}(X)$ is said to be a scalar-type spectral operator if there exists an equicontinuous spectral measure $P$ in $\mathscr{L}(X)$ and a $P$-integrable function $f$ such that $T=P(f)$. If $X$ is a Banach space, this agrees with the classical definition due to N. Dunford [7, XV, $\$ 4$ and XVII, §2]. If $T \in \mathscr{L}(X)$ is a scalar-type spectral operator for some equicontinuous spectral measure $P$ in $\mathscr{L}(X)$, and if $\mathscr{L}(X)$ is sequentially complete, then $T$ belongs to the closed algebra in $\mathscr{L}(X)$ generated by the $\mathscr{L}(X)$-closure, $\langle\mathscr{M}\rangle$, of the range of $P$ (see, for example, [22]). Since $\mathscr{M}$ is a Bade complete, equicontinuous Boolean algebra of projections in $X$ (see [5, Proposition 4.6]), we obtain the following result as an immediate consequence of Theorem 3.8 .

COROLlaRY 3.9. If $X$ is quasicomplete and if $\mathscr{L}(X)$ is sequentially complete, then every scalar-type spectral operator in $\mathscr{L}(X)$ is a reflexive operator.

Let us remark further that Theorem 3.8 may be sharpened if it is assumed in addition that $\mathscr{L}(X)$ is sequentially complete. Indeed, using Corollary 5.7 rather than Corollary 5.6 of [5], it follows easily that every closed unital subalgebra of $\langle\mathscr{M}\rangle$, is a reflexive subalgebra of $\mathscr{L}^{\sharp}(X)$.

For the case that the space $X$ is Banach, the result of Theorem 3.8 was proved by T. A. Gillespie [9, Theorem 4.6]. Gillespie's proof is based on an interesting factorization theorem for Banach function spaces. His method of proof does not seem to carry over to the locally convex setting, and the above proof of Proposition 3.2 is a simplification of the proof in the Banach space case. We like to point out, however, that from Gillespie's method a stronger result, at leasi for the case that $X$ is Banach, concerning the representation of linear functionals on $\langle\mathscr{M}\rangle$, can be deduced. For the sake of convenience we present the details in

Proposition 3.10. Let $\mathscr{M}$ be a Bade complete Boolean algebra of projections in the Banach space $X$, and let $\langle\mathscr{M}\rangle$ be the strongly closed subalgebra of $\mathscr{L}(X)$ generated by $\mathscr{M}$. Then any $\varphi \in\langle\mathscr{M}\rangle_{n}^{\tilde{n}}$ is of the form $\varphi=x \otimes x^{\prime}$ with $x \in X, x^{\prime} \in X^{\prime}$. Equivalently, $\langle\mathscr{M}\rangle_{n}^{\tilde{n}}=\langle\mathscr{M}\rangle^{\prime}$.

Proof. Let $0 \leqslant \varphi \in\langle\mathscr{M}\rangle_{n}^{\tilde{n}}$ be given and let $C_{\varphi}$ be the carrier of $\varphi$ in $\langle\mathscr{M}\rangle$. There exists $E_{0} \in \mathscr{M}$ such that $C_{\varphi}=E_{0}\langle\mathscr{M}\rangle$. Let $\left\{E_{\alpha}\right\}$ be a maximal disjoint system of 
carrier projections in $C_{\varphi}$. Since $\varphi$ is strictly positive on $C_{\varphi}$, it follows that this system $\left\{E_{\alpha}\right\}$ is at most countable, $\left\{E_{n}\right\}_{n=1}^{\infty}$ say, corresponding to elements $\left\{x_{n}\right\}_{n=1}^{\infty}$. Note that $E_{0}=\bigvee_{n=1}^{\infty} E_{n}$. We can assume that $\left\|x_{n}\right\|=1$ for all $n$. Define $x_{0}=\sum_{n=1}^{\infty} 2^{-n} x_{n}$, and observe that $E_{0}=E x_{0}$. This implies that $\varphi(E)=0$ for all $E \in \mathscr{M}$ with $E x_{0}=0$. Indeed, if $E x_{0}=0$, then $(I-E) x_{0}=x_{0}$, so $E_{0} \leqslant I-E$ and hence $E_{0} \wedge E=0$, which implies $E \in N_{\varphi}$ and therefore $\varphi(E)=0$.

Now let $\mathscr{M}$ be displayed as the range of a closed equicontinuous spectral measure $P$ on the Borel sets $\mathscr{B}$ of the Stone space of $\mathscr{M}$. Then $\varphi$ corresponds to a complex measure $\nu$ on $\mathscr{B}$ which satisfies $\nu \ll P x_{0}$, i.e., $\nu(B)=0$ whenever $\left(P x_{0}\right)(B)=0$. Now it follows from [9, Theorem 4.2] that there exists $x_{0}^{\prime} \in X^{\prime}$ such that $\nu(B)=$ $\left\langle\left(P x_{0}\right)(B), x_{0}^{\prime}\right\rangle$ for all $B \in \mathscr{B}$. Thus $\varphi(E)=\left\langle E x_{0}, x_{0}^{\prime}\right\rangle$ for all $E \in \mathscr{M}$. The order conntinuity of $\varphi$, combined with the Freudenthal spectral theorem, now shows that $\varphi(T)=\left\langle T x_{0}, x_{0}^{\prime}\right\rangle$ for all $T \in \mathscr{M}$, and hence $\varphi=x_{0} \otimes x_{0}^{\prime}$.

The point of the above proposition is that any order continuous normal linear functional on $\langle\mathscr{M}\rangle$ is strongly continuous, and this goes back to $\mathrm{R}$. Pallu de la Barriére [20] in the case that $\langle\mathscr{M}\rangle$ is an abelian $W^{*}$-algebra. It seems to be an interesting question whether the above result is true in the general locally convex setting.

4. Remarks on the spectral resolution. If $T$ is a scalar-type spectral operator in the quasicomplete space $X$, then there exists a unique equicontinuous, $\mathscr{L}(X)$-valued spectral measure $Q$ defined on the Borel subsets of the complex plane $\mathbf{C}$ such that the identity function on $\mathbf{C}$ is $Q$-integrable and $T=\int_{\mathbf{C}} z d Q(z)$; this follows from [19, Propositions 1.2.1, 1.2.7]. This unique spectral measure $Q$ is called the resolution of the identity of $T$. We now remark that the range of the resolution of the identity of $Q$ is precisely the Boolean $\sigma$-subalgebra generated by the Freudenthal system of $T$ in any strongly closed algebra $\langle\mathscr{M}\rangle$ containing $T$ which is generated by an equicontinuous Bade-complete Boolean algebra $\mathscr{M}$. We indicate the details.

Let $\mathscr{M}$ be an equicontinuous, Bade complete Boolean algebra of projections in the quasicomplete space $X$ and let $\langle\mathscr{M}\rangle$ be the strongly closed subalgebra of $\mathscr{L}(X)$ generated by $\mathscr{M}$. Let $T$ be a given element of $\langle\mathscr{M}\rangle$ and write $T=\operatorname{Re} T+i \operatorname{Im} T$ with $\operatorname{Re} T, \operatorname{Im} T \in \operatorname{Re}\langle\mathscr{M}\rangle$. We denote by $\{E(\lambda): \lambda \in \mathbf{R}\},\{F(\lambda): \lambda \in \mathbf{R}\}$ the (Freudenthal) spectral systems of $\operatorname{Re} T, \operatorname{Im} T$ in the Dedekind complete Riesz space $\operatorname{Re}\langle\mathscr{M}\rangle$ with respect to the weak order unit $I$. For basic properties of these spectral systems, the reader is referred to $[\mathbf{1 8}, \S 38]$. The Freudenthal spectral system $\{G(z)$ : $z \in \mathbf{C}\}$ of $T$ in the complex Riesz space $\langle\mathscr{M}\rangle$ is now defined by setting $G(z)=$ $E(\lambda) F(u)$ if $z=\lambda+i \mu, \lambda, \mu \in \mathbf{R}$. It is a routine task to see that the spectral system $\{G(z): z \in \mathbf{C}\}$ induces, in the obvious manner, a countably-additive, $\mathscr{M}$-valued multiplicative set function $Q_{0}$ defined on the ring generated by the usual collection of cells in $\mathbf{R}^{2}$, considered as subsets of $\mathbf{C}$. Since $\langle\mathscr{M}\rangle$ is Dedekind complete and has Lebesgue topology it follows from [1, Theorem 21.1] that $\langle\mathscr{M}\rangle$ has weakly compact order intervals and hence, by the Kluvanek extension theorem [11, p. 178] (see also [28, $\S 5,6$ of Chapter XI]), it follows that $Q_{0}$ extends to a countably-additive measure, denoted again by $Q_{0}$, on the Borel subsets of $\mathbf{C}$. Further, it is not difficult to show that $Q_{0}$ is an $\mathscr{M}$-valued spectral measure (see [6, 
Proposition 3.6]). The Freudenthal spectral theorem now implies that the identity function on $\mathbf{C}$ is $Q_{0}$-integrable and $T=\int_{\mathbf{C}} z d Q_{0}(z)$. It follows that $T$ is a scalar-type spectral operator and that the spectral measure $Q_{0}$ is precisely the resolution of the identity for $T$. Moreover, the range of the spectral resolution of $T$ is precisely the Boolean $\sigma$-subalgebra of $\mathscr{M}$ generated by the Freudenthal system of $T$ relative to $I$ in the Riesz space $\langle\mathscr{M}\rangle$.

Suppose now that $T$ is a scalar-type spectral operator in the quasicomplete space $X$ with spectral resolution $Q$. If $T^{*}=\int_{\mathbf{C}} \bar{z} d Q(z)$, then it is clear that $I, T$ and $T^{*}$ belong to the strongly closed subalgebra of $\mathscr{L}(X)$ generated by the range of the spectral resolution $Q$ of $T$. Of course, if $X$ is a Hilbert space and if $T$ is normal, then it is a classical result that the strongly closed subalgebra of $\mathscr{L}(X)$ generated by $I, T$ and $T^{*}$ coincides with the strongly closed subalgebra of $\mathscr{L}(X)$ generated by the range of the spectral resolution of $T$. This result is still valid if $X$ is Banach, but fails in general. We present first an example of this pathology, which finds its roots in the work of Stieltjes [26].

EXAmple 4.1. Let $X$ be the space of all Lebesgue measurable functions $f$ on $\mathbf{R}$ for which

$$
\int_{\mathbf{R}}|f(x)|\left(1+|x|^{n}\right) d x<\infty, \quad n=0,1,2, \ldots
$$

With respect to the pointwise operations, $X$ is a Dedekind complete complex Riesz space. We endow $X$ with the $|\sigma|\left(X, \tilde{X_{n}}\right)$-topology [1, Definition 6.5], which is a complete Lebesgue topology. Note that $\tilde{X_{n}}$ can be identified with the Köthe dual (associate ideal) of $X$, as $X$ is an ideal of measurable functions [30, $\S 86]$. Let $\mathscr{M}$ be the Boolean algebra of all band projections in $X$. By Proposition 2.4, $\langle\mathscr{M}\rangle$ is the space of all orthomorphisms in $X$, which can be identified as well with an ideal of measurable functions. Note that all polynomials are contained in $\langle\mathscr{M}\rangle$. Define $T \in\langle\mathscr{M}\rangle$ by setting $(T f)(x)=x f(x), x \in \mathbf{R}$. It is clear that $T$ is a scalar-type spectral operator and the range of the resolution of the identity is precisely $\mathscr{M}$. Further, it is clear that $T=T^{*}$. Assume that $\mathscr{M}$ is contained in the strongly closed subalgebra generated by $I$ and $T$, which we denote by $\langle I, T\rangle$. Then, in fact, $\langle\mathscr{M}\rangle=\langle I, T\rangle$. Let $S \in\langle\mathscr{M}\rangle$ be defined by

$$
(S f)(x)=\sin (2 \pi \ln |x|) f(x), \quad x \in \mathbf{R},
$$

for all $f \in X$. Since $S \in\langle I, T\rangle$, it follows that, for each $0 \leqslant \varphi \in \tilde{X_{n}}$ and $0 \leqslant f \in X$, there exists a sequence $\left\{p_{n}\right\}_{n=1}^{\infty}$ of polynomials such that

$$
\varphi\left(\left|p_{n}(T)-S\right| f\right) \rightarrow 0 \quad \text { as } n \rightarrow \infty .
$$

Now if $f \in X$ is defined by setting $f(x)=|x|^{-\ln |x|}, x \in \mathbf{R}$, and if $\varphi$ is Lebesgue integration over $\mathbf{R}$, then, for the corresponding sequence of polynomials, we have that

$$
\int_{\mathbf{R}}\left|p_{n}(x)-\sin (2 \pi \ln |x|)\right||x|^{-\ln |x|} d x \rightarrow 0
$$


as $n \rightarrow \infty$. However, since

$$
\int_{\mathbf{R}} x^{k} \sin (2 \pi \ln |x|)|x|^{-\ln |x|} d x=0, \quad k=0,1, \ldots,
$$

it follows that

$$
\int_{\mathbf{R}} p_{n}(x) \sin (2 \pi \ln |x|)|x|^{-\ln |x|} d x=0, \quad n=1,2, \ldots,
$$

and therefore (17) implies that

$$
\int_{\mathbf{R}}\{\sin (2 \pi \ln |x|)\}^{2}|x|^{-\ln |x|} d x=0
$$

which is clearly a contradition. It follows that the range of the spectral resolution of $T$ is not contained in the strongly closed algebra generated by $I$ and $T$.

We remark that Example 4.1 shows, in conjunction with Theorem 3.8, that it is possible for a real scalar-type spectral operator to have more invariant subspaces than the range of its spectral resolution. On the other hand, we now exhibit a wide class of spaces for which this pathology does not occur.

Once again, let $\mathscr{M}$ be a Bade complete Boolean algebra of projections in the quasicomplete space $X$ and let $\langle\mathscr{M}\rangle$ be the strongly closed subalgebra of $\mathscr{L}(X)$ generated by $\mathscr{M}$. If $T$ is any element of $\langle\mathscr{M}\rangle$, then $T$ is scalar-type spectral and we denote the spectral resolution of $T$ by $Q$. Once again, set $T^{*}=\int_{\mathrm{C}} \bar{z} d Q(z)$ and denote the strongly closed subalgebra of $\mathscr{L}(X)$ generated by $I, T$ and $T^{*}$ by $\left\langle I, T, T^{*}\right\rangle$. The first observation that we make is that the range, $R(Q)$, of the spectral resolution $Q$ is contained in $\left\langle I, T, T^{*}\right\rangle$ if and only if $\left\langle I, T, T^{*}\right\rangle$ is a Riesz subspace of $\langle\mathscr{M}\rangle$. In fact, if $R(Q)$ is contained $\left\langle I, T, T^{*}\right\rangle$, then $\left\langle I, T, T^{*}\right\rangle$ is equal to the strongly closed subalgebra of $\langle\mathscr{M}\rangle$ generated by $R(Q)$, and hence $\left\langle I, T, T^{*}\right\rangle$ is a Riesz subspace of $\langle\mathscr{M}\rangle$. Now if we assume that $\left\langle I, T, T^{*}\right\rangle$ is a Riesz subspace of $\langle\mathscr{M}\rangle$, then it is not difficult to see that $\left\langle I, T, T^{*}\right\rangle$ is in fact a Dedekind complete Riesz space in its own right and that the induced topology in $\left\langle I, T, T^{*}\right\rangle$ is Lebesgue. It now follows from the uniqueness of the Freudenthal spectral system [18, Theorem 40.8] in the space $\langle\mathscr{M}\rangle$ that $R(Q)$ is in fact contained in $\left\langle I, T, T^{*}\right\rangle$. The above observation, combined with the fact [10, Theorem 6.3] that a uniformly closed, conjugate closed unital subalgebra of a space $C(\Omega)$ is a Riesz subspace (where $C(\Omega$ ) denotes the space of all complex continuous functions on the completely regular Hausdorff space $\Omega$ ), yields the following remarkable result.

Proposition 4.2. Let $\mathscr{M}$ be an equicontinuous Bade complete Boolean algebra of projections in the quasicomplete space $X$. Suppose that the strongly closed subalgebra $\langle\mathscr{M}\rangle$ of $\mathscr{L}(X)$ generated by $\mathscr{M}$ is algebraically isomorphic to $C(\Omega)$ for some completely regular Hausdorff space $\Omega$. Then, for all $T \in\langle\mathscr{M}\rangle$, the range of the spectral resolution $Q$ of $T$ is contained in the strongly closed subalgebra of $\mathscr{L}(X)$ generated by $I, T$ and $T^{*}$.

We remark that if $X$ is Banach, then it is well known that $\langle\mathscr{M}\rangle$ is isomorphic to $C(\Omega)$ for some compact Hausdorff space $\Omega$, and so the conclusion of the preceding proposition holds. 
In view of Example 4.1, it seems natural to describe intrinsically the strongly closed subalgebra of $\mathscr{L}(X)$ generated by the range of the spectral resolution of a given scalar-type spectral operator $T$, in terms of $T$ and its conjugate $T^{*}$. We show first that such algebras are inverse closed, a result which is interesting in its own right and which was observed in [23, Lemma 3]. The following result shows that even more is true. We denote by $\mathscr{L}^{\sharp}(X)$ the space of all everywhere defined linear mappings on $X$.

Proposition 4.3. Let $X$ be quasicomplete and assume that $\mathscr{L}(X)$ is sequentially complete. Let $\mathscr{M}$ be an equicontinuous Bade complete Boolean algebra of projections in $X$ and let $\langle\mathscr{M}\rangle$ be the strongly closed subalgebra of $\mathscr{L}(X)$ generated by $\mathscr{M}$. If $T \in\langle\mathscr{M}\rangle$ and if $T$ is invertible in $\mathscr{L}^{\sharp}(X)$, then $T^{-1}$ is necessarily continuous and $T^{-1} \in\langle\mathscr{M}\rangle$.

Proof. We show first that $|T|$ is a weak order unit in the Dedekind complete Riesz space $\langle\mathscr{M}\rangle$. In fact, suppose that $0 \leqslant S \in\langle\mathscr{M}\rangle$ and $|T| \wedge S=0$ in $\langle\mathscr{M}\rangle$. It follows from [5, Proposition 4.1] that $|T x| \wedge S x=0$ in the Riesz space $\mathscr{M}[x]$ for all $x \in X$. If $x \in X$ is given, then $x$ is a weak unit in $\mathscr{M}[x]$ and so $S x \wedge n x \uparrow_{n} S x$. It follows that $|T|(S x \wedge n x) \uparrow_{n}|T|(S x)$. Since $|T|(S x \wedge n x) \leqslant|T|(n x)=n|T|(x)$, it follows that $|T|(S x \wedge n x) \perp S x$ for $n=1,2 \ldots$ and hence $|T|(S x) \perp S x$. However, since the restriction of $|T|$ to $\mathscr{M}[x]$ is an orthomorphism of $\mathscr{M}[x]$ by [5, Proposition 4.1], it follows that $|T|(S x) \perp|T|(S x)$ and so $|T|(S x)=|T|(S x)=0$. Since $T$ is injective, it follows that $S x=0$ for all $x \in X$ and so $S=0$.

Let now $E_{n} \in \mathscr{M}$ be the component of the identity in the band in $\langle\mathscr{M}\rangle$ generated by $(|T|-I / n)^{+}, n=1,2, \ldots$ Since $(|T|-I / n)^{+} \uparrow_{n}|T|$, and since $|T|$ is a weak order unit in $\langle\mathscr{M}\rangle$, it follows that $E_{n} \uparrow_{n} I$. Moreover, since $(|T|-I / n) E_{n}=$ $(|T|-I / n)^{+} \geqslant 0$, it follows that $|T| E_{n} \geqslant E_{n} / n, n=1,2, \ldots$ It follows from [30, Theorem 146.3], that $|T| E_{n}+\left(I-E_{n}\right)$ is invertible in $\langle\mathscr{M}\rangle$ for $n=1,2, \ldots$ and consequently $T E_{n}+\left(I-E_{n}\right)$ is invertible in $\langle\mathscr{M}\rangle$ for $n=1,2, \ldots$ It is easy to see that $\left(T E_{n}+I-E_{n}\right)^{-1}=T^{-1} E_{n}+\left(I-E_{n}\right)$ and so $T^{-1} E_{n} \in \mathscr{M}$ for $n=1,2, \ldots$ From the fact that $T$ is surjective, it follows easily that $\left(T^{-1} E_{n}\right)(x) \rightarrow T^{-1}(x)$ for all $x \in X$, and the result follows.

A subalgebra $\mathscr{A}$ of $\mathscr{L}(X)$ is called inverse closed if whenever $T \in \mathscr{A}$ and $T$ is invertible in $\mathscr{L}(X)$, it follows that $T^{-1} \in \mathscr{A}$. Once again, for any scalar-type spectral operator $T$ in the quasicomplete space $X$ with spectral resolution $Q$, we write $T^{*}=\int_{\mathbf{C}} \bar{z} d Q(z)$. We may now state the final result of this paper.

THEOREM 4.4. Let $T$ be a scalar-type spectral operator in the quasicomplete space $X$. Assume that $\mathscr{L}(X)$ is sequentially complete. The strongly closed subalgebra of $\mathscr{L}(X)$ generated by the range of the spectral resolution of $T$ is precisely the smallest strongly closed, inverse closed subalgebra of $\mathscr{L}(X)$ containing $I, T$ and $T^{*}$.

Proof. If $\mathscr{M}$ is the closure of the range of the spectral resolution of $T$, then $\mathscr{M}$ is an equicontinuous, Bade complete Boolean algebra of projections in $\mathscr{L}(X)[\mathbf{5}$, Proposition 4.6] and consequently the strongly closed subalgebra $\langle\mathscr{M}\rangle$ of $\mathscr{L}(X)$ is precisely the strongly closed subalgebra of $\mathscr{L}(X)$ generated by the range of the 
spectral resolution of $T$. Observe that $I, T$ and $T^{*}$ are elements of $\langle\mathscr{M}\rangle$ and that $\langle\mathscr{M}\rangle$ is inverse closed by Proposition 4.3. Therefore, denoting the smallest inverse closed strongly closed subalgebra of $\mathscr{L}(X)$ containing $I, T$ and $T^{*}$ by $\mathbf{A}$, it follows that $\mathbf{A} \subseteq\langle\mathscr{M}\rangle$. It is clear that $\operatorname{Re} T$ and $\operatorname{Im} T$ belong to $\mathbf{A}$. Denote by $\operatorname{Re} \mathbf{A}$ the set of all $S \in \mathbf{A}$ for which $S=S^{*}$. Observe that $\operatorname{Re} \mathbf{A}$ is a strongly closed subalgebra of $\operatorname{Re}\langle\mathscr{M}\rangle$, containing $I, \operatorname{Re} T$ and $\operatorname{Im} T$. Moreover, $\operatorname{Re} \mathbf{A}$ is inverse closed in $\mathscr{L}(X)$ and hence in $\langle\mathscr{M}\rangle$. We assert that $\operatorname{Re} \mathbf{A}$ is a $\operatorname{Riesz}$ subspace of $\operatorname{Re}\langle\mathscr{M}\rangle$. In fact, suppose $S \in \operatorname{ReA}$. Since $I+S^{2} \geqslant I$, it follows that $\left(I+S^{2}\right)^{-1}$ exists in $\langle\mathscr{M}\rangle$ and hence $\left(I+S^{2}\right)^{-1} \in \operatorname{Re} \mathbf{A}$. If now $A=S\left(I+S^{2}\right)^{-1}$, then $A \in \operatorname{Re} \mathbf{A},|A| \leqslant I$ holds in $\mathscr{M}$. This implies that there exists a sequence $\left\{p_{n}\right\}_{n=1}^{\infty}$ of polynomials with real coefficients, such that $p_{n}(A) \rightarrow|A|, I$-uniformly in $\langle\mathscr{M}\rangle$. This implies in particular that $p_{n}(A) \rightarrow|A|$ strongly and since $p_{n}(A) \in \operatorname{Re} \mathbf{A}$ for all $n=1,2, \ldots$, it follows that $|A| \in \operatorname{Re} \mathbf{A}$. Thus, $\operatorname{Re} \mathbf{A}$ is a $\operatorname{Riesz}$ subspace of $\operatorname{Re}\langle\mathscr{M}\rangle$. Since $\operatorname{Re}\langle\mathscr{M}\rangle$ is a Dedekind complete Riesz space with Lebesgue locally solid topology, and since $\operatorname{Re} A$ is a closed Riesz subspace, it follows easily that $\operatorname{Re} \mathbf{A}$ is a Dedekind complete space in its own right and that the induced topology is Lebesgue. From the uniqueness of the (Freudenthal) spectral system, it follows that the spectral systems in $\langle\mathscr{M}\rangle$ of $\operatorname{Re} T$ and $\operatorname{Im} T$ are contained in $\operatorname{Re} \mathbf{A}$ and hence the Freudenthal spectral system of $T$ is contained in $\operatorname{Re} \mathbf{A}$. Since $\mathscr{M}$ is equal to the strong closure of the spectral system of $T$, we may conclude that $\mathscr{M} \subseteq \operatorname{Re} \mathbf{A}$ and hence $\langle\mathscr{M}\rangle \subseteq \mathbf{A}$. It follows that $\mathrm{A}=\langle\mathscr{M}\rangle$ and the proof is complete.

\section{REFERENCES}

1. C. D. Aliprantis and O. Burkinshaw, Locally solid Riesz spaces, Academic Press, New York, 1978.

2. W. G. Bade, On Boolean algebras of projections and algebras of operators, Trans. Amer. Math. Soc. 80 (1955), 345-359.

3. E. Berkson and H. R. Dowson, On reflexive scalar-type spectral operators, J. London Math. Soc. (2) 8 (1974), 652-656.

4. F. Beukers, C. B. Huijsmans and B. de Pagter, Unital embedding and complexification of f-algebras, Math. Z. 183 (1983), 131-144.

5. P. G. Dodds and B. de Pagter, Orthomorphisms and Boolean algebras of projections, Math. Z. 187 (1984), 361-381.

6. P. G. Dodds and W. Ricker, Spectral measures and the Bade reflexivity theorem, J. Funct. Anal. 61 (1985), 136-163.

7. N. Dunford and J. T. Schwartz, Linear operators. Part III: Spectral operators, Wiley-Interscience, New York, 1971.

8. T. A. Gillespie, Cyclic Banach spaces and reflexive operator algebras, Proc. Roy. Soc. Edinburgh Sect. A 78 (1978), 225-235.

9. B__ Boolean algebras of projections and reflexive algebras of operators, Proc. London Math. Soc. 37 (1978), 56-74.

10. C. B. Huijsmans and B. de Pagter, Subalgebras and Riesz subspaces of an f-algebra, Proc. London Math. Soc. 48 (1984), 161-174.

11. I. Kluvanek, The extension and closure of vector measures, Vector and Operator-Valued Measures and Applications, Academic Press, New York, 1973, pp. 175-189.

12. Conical measures and vector measures, Ann. Inst. Fourier (Grenoble) 27 (1977), 83-105.

13. I. Kluvanek and G. Knowles, Vector measures and control systems, North-Holland, Amsterdam, 1976.

14. D. R. Lewis, Integration with respect to vector measures, Pacific J. Math. 33 (1970), 157-165.

15. __ On integration and summability in vector spaces, Illinois J. Math. 16 (1972), 294-307.

16. J. Lindenstrauss and L. Tzafriri, Classical Banach spaces. II. Function spaces, Ergeb. Math. Grenzgeb., Band 97, Springer-Verlag, Berlin, Heidelberg and New York, 1979. 
17. W. A. J. Luxemburg, Some aspects of the theory of Riesz spaces, Univ. of Arkansas Lecture Notes in Math. 4, Fayetteville, 1979.

18. W. A. J. Luxemburg and A. C. Zaanen, Riesz spaces. I, North-Holland, Amsterdam, 1971.

19. F. Maeda, Spectral theory in locally convex spaces, Ph. D. Thesis, Yale University, 1961.

20. R. Pallu de la Barrière, Sur les algèbres d'opérateurs dans les espaces hilbertiens, Bull. Soc. Math. France 82 (1954), 1-51.

21. C. Rall, Über Boolesche Algebren von Projektionen, Math. Z. 153 (1977), 199-217.

22. W. Ricker, On Boolean algebras of projections and scalar-type spectral operators, Proc. Amer. Math. Soc. 87 (1983), 73-77.

23. Integral Equations Operator Theory 8 (1985), 276-288.

24. H. H. Schaefer, Spectral measures in locally convex algebras, Acta Math. 107 (1962), 125-173.

25. __ Topological vector spaces, Springer-Verlag, Berlin, Heidelberg and New York, 1971.

26. T. J. Stieltjes, Recherches sur les fraction continues, Ann. Fac. Sci. Univ. Toulouse 8 (1894), T1-122; 9 (1895), A5-97.

27. A. I. Veksler, Cyclic Banach spaces and Banach lattices, Soviet Math. Dokl. 14 (1973), 1773-1779.

28. B. Z. Vulikh, Introduction to the theory of partially ordered spaces, Wolters-Noordhoff, Groningen, 1967.

29. B. Walsh, Structure of spectral measures on locally convex spaces, Trans. Amer. Math. Soc. 120 (1965), 295-326.

30. A. C. Zaanen, Riesz spaces. II, North-Holland, Amsterdam, 1983.

School of Mathematical Sciences, The Flinders University of South Australia, Bedford Park, S.A. 5042, Australia (Current address of P. G. Dodds)

Department of Mathematics, Delft University of Technology, Delft, The Netherlands (Current address of B. de Pagter)

Department of Mathematics, I.A.S., Australian National University, Canberra, A.C.T. 2600, Australia

Current address (W. Ricker), School of Mathematics and Physics, Macquarie University, North Ryde, 2113, Australia 